\title{
Gravity currents and internal waves in a stratified fluid
}

\author{
BRIAN L. WHITE ${ }^{1}$ AND KARL R. HELFRICH \\ ${ }^{1}$ Department of Marine Sciences, University of North Carolina at Chapel Hill, Chapel Hill, NC, USA \\ bwhite@unc.edu \\ ${ }^{2}$ Department of Physical Oceanography, Woods Hole Oceanographic Institution, Woods Hole, MA, USA \\ khelfrich@whoi.edu
}

(Received 9 October 2007 and in revised form 11 August 2008)

A steady theory is presented for gravity currents propagating with constant speed into a stratified fluid with a general density profile. Solution curves for front speed versus height have an energy-conserving upper bound (the conjugate state) and a lower bound marked by the onset of upstream influence. The conjugate state is the largestamplitude nonlinear internal wave supported by the ambient stratification, and in the limit of weak stratification approaches Benjamin's energy-conserving gravity current solution. When the front speed becomes critical with respect to linear long waves generated above the current, steady solutions cannot be calculated, implying upstream influence. For non-uniform stratification, the critical long-wave speed exceeds the ambient long-wave speed, and the critical-Froude-number condition appropriate for uniform stratification must be generalized. The theoretical results demonstrate a clear connection between internal waves and gravity currents. The steady theory is also compared with non-hydrostatic numerical solutions of the full lock release initialvalue problem. Some solutions resemble classic gravity currents with no upstream disturbance, but others show long internal waves propagating ahead of the gravity current. Wave generation generally occurs when the stratification and current speed are such that the steady gravity current theory fails. Thus the steady theory is consistent with the occurrence of either wave-generating or steady gravity solutions to the dam-break problem. When the available potential energy of the dam is large enough, the numerical simulations approach the energy-conserving conjugate state. Existing laboratory experiments for intrusions and gravity currents produced by full-depth lock exchange flows over a range of stratification profiles show excellent agreement with the conjugate state solutions.

\section{Introduction}

The behaviour of a gravity current propagating in a stratified fluid presents an opportunity to revisit a classic problem of fluid mechanics that is highly relevant to environmental flows. In the classic gravity current problem (Benjamin 1968; Simpson 1999), a mass of dense (or light) fluid propagates into a lighter (heavier) ambient fluid until arrested by friction or boundaries. Motion can be initiated by the removal of a barrier separating light and dense reservoirs, known as a lock exchange. Benjamin (1968) found solutions for steady gravity current propagation speeds, including a special energy-conserving solution when the current occupies exactly half the fluid depth. Smaller current heights imply energy dissipation and larger heights require negative dissipation (energy input). Several extensions have been considered, including non-Bousinesq currents (Lowe, Rottman \& Linden 2005) and partial depth lock 


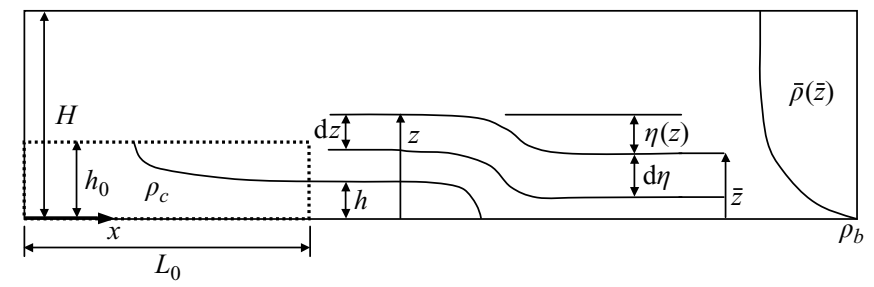

FIgURE 1. Problem description. Gravity current and ambient properties: gravity current height $h$, layer depth $H$, initial dam height $h_{o}$, and length $L_{o}$, isopycnal displacement $\eta$, ambient density profile $\bar{\rho}(\bar{z})$, where $\bar{z}=z-\eta(z)$, and top, $\rho_{t}$, and bottom, $\rho_{b}$, densities.

releases for which the initial-value problem gives energy-conserving gravity currents different from Benjamin's energy-conserving solution (Shin, Dalziel \& Linden 2004). Although, this latter extension has been been challenged by Ungarish (2008).

The presence of ambient stratification allows for a richer set of behaviours than in unstratified gravity currents because the fast long internal waves can propagate in the fluid ahead of the current. These long internal waves are widespread in the stratified ocean and atmosphere. Atmospheric fronts generate undular bores such as the Morning Glory in northern Australia (Rottman \& Simpson 1989), which steepen to produce large-amplitude solitary-type waves. Buoyant river plumes have been observed to generate nonlinear internal waves in the coastal ocean (Nash \& Moum 2005). The advection of deep cold-water masses by the internal tide causes internal bores which steepen to form internal solitary waves (Pineda 1991). These nonlinear internal waves closely resemble those generated by the interaction of the barotropic tide with topography (see review by Helfrich \& Melville 2006), and may result in significant ocean mixing (Nash \& Moum 2005) and transport of marine organisms (Helfrich \& Pineda 2003).

A depiction of the problem considered is shown in figure 1. A volume of fluid with height $h_{o}$, length $L_{o}$ and density $\rho_{c}$ collapses into an ambient region with general stratification $\bar{\rho}(\bar{z})$, resulting in a gravity current with thickness $h$ and speed $U$. The current displaces isopycnals a distance $\eta$, directly by forcing them upward as it flows beneath, and indirectly by exciting waves which propagate ahead. The flow is confined to a layer of finite depth, $H$, with top and bottom densities $\rho_{t}$ and $\rho_{b}$. After sufficient time following the dam-break, the current becomes long compared with $H$, and the flow is nearly uniform (independent of $x$ ) in the region above the current. The isopycnal displacement between the region upstream (in front) of the current to the region above it may then be approximated as a one-dimensional function, $\eta(z)$. We will refer to the region in front of the current as upstream because in the moving reference frame the problem is similar to stratified flow over topography, and this terminology maintains consistency with upstream influence problems. Also note that for the free-slip bottom condition that we consider, the dense bottom current is analogous under the Boussinesq approximation to a surface current with an equivalent buoyancy deficit. Moreover, by symmetry, when $\rho_{c}=\rho_{b}$, the bottom current is equivalent to a neutral density intrusion (Ungarish 2005b; Flynn \& Linden 2006) into a stratified fluid symmetric around $z=0$.

The stratification in the ambient fluid supports waves with a linear long-wave speed, $c_{o}$, that depends on $\bar{\rho}(\bar{z})$. Nash \& Moum (2005) observed internal waves in the Columbia River plume when the front speed was subcritical to the long-wave speed, $U<c_{o}$. Initially, the plume travelled faster than $c_{o}$, behaving as a surface 
gravity current, but as it slowed, large-amplitude waves formed near the front and propagated ahead of it. In laboratory experiments of gravity currents with linear background stratification, Maxworthy et al. (2002) also observed internal waves in the subcritical regime, which were generated at the front and carried dense fluid ahead of the current. In the supercritical regime, there were no upstream waves and the gravity currents resembled the solutions of Benjamin (1968).

Ungarish \& Huppert (2002) introduced a shallow-water hydraulic model for a gravity current moving at the base of a stratified ambient and their results agreed with the experiments of Maxworthy et al. (2002). They found the speed is driven by the pressure gradient at the mid-height of the nose, which is modified by ambient stratification, but the Froude-number condition relating speed to pressure gradient is roughly independent of stratification. Ungarish (2005a) extended the shallow-water theory to include nonlinear stratification and Ungarish (2005b) used the theory to predict neutral density intrusions propagating into a mixed region between two homogeneous layers. Results for the front speed agreed well with experiments by Faust \& Plate (1984) and Britter \& Simpson (1981). In light of its good agreement with experiments, the shallow-water model appears to be a robust tool for predicting gravity current behaviour in stratified fluids. However, drawbacks include the requirement of an empirical Froude-number condition (Ungarish \& Huppert 2002) and the inherent neglect of internal waves generated in the ambient fluid. Remarkably, Ungarish \& Huppert (2006) found that very little energy is exchanged between the waves and the current, even as the waves slow down the front under subcritical conditions. Nonetheless, we are concerned with the wave behaviour, and therefore must move beyond the shallow-water model.

Ungarish (2006) developed a theory for the steady gravity current speed in a linearly stratified ambient. He went beyond the shallow-water model by using Long's method (Baines 1997) to compute the displacement of isopycnals as they move over the current. The theory illustrates the effect of ambient stratification on propagation speed and energy dissipation, and front speeds approach the classic Benjamin (1968) results in the limit of weak ambient stratification. Some stratification configurations have multiple steady solutions, corresponding to discrete internal modes from Long's model, with higher modes having slower associated gravity current speeds. Ungarish suggested that a current released by dam break will lock onto the slowest stable solution and some support is given by numerical results of Birman, Meiburg \& Ungarish (2007) showing correspondence between front speed and second-mode solutions for subcritical gravity currents. However, Long's method does not give higher-mode solutions for all combinations of $\rho_{c}$ and $h$, and because it is a steady theory, is not strictly valid for subcritical currents which generate upstream waves. Thus, the higher-mode solutions are an open question and one we explore in this paper.

To illustrate the dichotomy between wave and gravity-current behaviour, the results from a continuously stratified non-hydrostatic numerical model for the initial-value problem with two different dam heights, $h_{o} / H=0.6$ and $h_{o} / H=0.9$, and a linearly stratified ambient density profile, are shown in figure 2 . These results are described in greater detail in subsequent sections. For now, note the distinction between the first case, with waves generated at the front and outrunning the gravity current, and the second, which resembles a classic Benjamin gravity current, with a steady propagation speed and no waves. The increase in $h_{o}$ from cases $(a)$ to $(b)$ represents an increase in the initial available potential energy which produces a larger, faster current. In $(a)$, the current is subcritical with respect to the linear long-wave speed, $U / c_{o}=0.75$, whereas in $(b)$ the current is nearly critical $U / c_{o}=0.95$. 

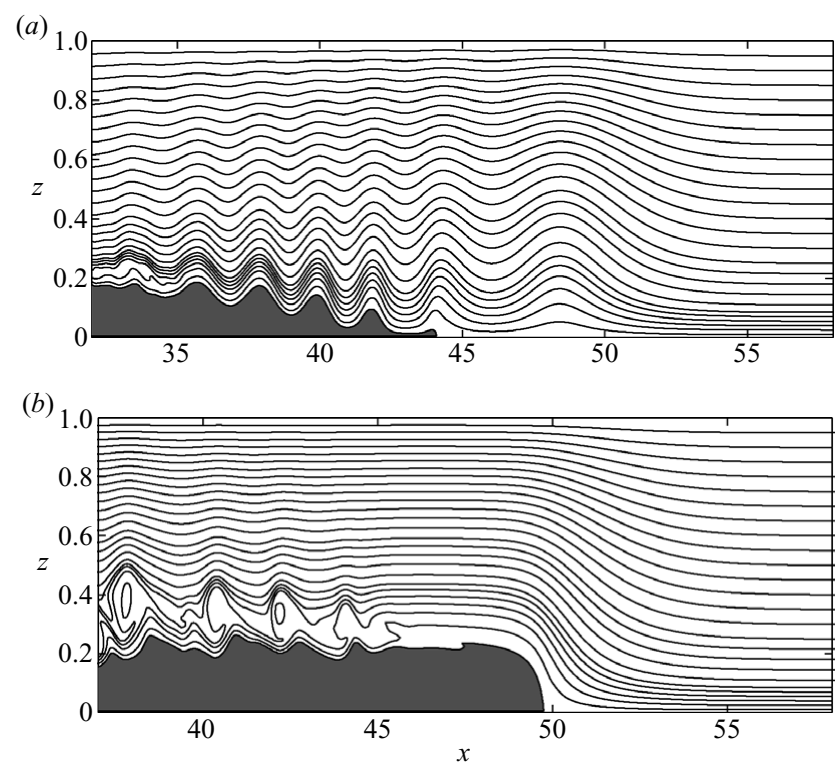

Figure 2. Comparison between two numerical solutions to the dam-break problem with different initial dam height $(S=1)$. (a) $h_{o}=0.6,(b) h_{o}=0.9$. The first is subcritical, with internal wave generation; the second is nearly critical, with a classic gravity-current appearance. The lock is at $x=20$. The total depth, $H$, is used to scale $x, z$, and the lock depth $h_{o}$.

We begin by extending the steady theory of Ungarish (2006) to an ambient fluid with a general density profile, and we identify a special energy-conserving solution for each stratification which is analogous to Benjamin's classic solution. Bounds on the steady theory predict the conditions for either steady gravity currents or gravity currents that generate internal waves in two-dimensional Navier-Stokes simulations of the initial dam-break problem. The numerical solutions show that the energy-conserving solutions are approached as the height of the dam (i.e. the available potential energy) is increased. In $\S 2$, governing equations and non-dimensional variables are introduced. In $\S 3$, literature on nonlinear internal waves is summarized to illustrate the connection with gravity currents in a stratified fluid. In $\S \S 4$ and 5 , theory for steady gravity currents in general stratification is developed and solutions are described. In $\S 6$, numerical calculations of the dam-break initial-value problem are described and compared with the steady theory to develop bounds on steady gravity current and internal wave-generating solutions. Finally, in $\S 7$, the steady theory is compared with experimental results on gravity currents and intrusions in stratified fluids available from the general literature.

\section{Governing equations and non-dimensionalization}

A general description of internal waves and gravity currents in a stratified fluid begins with the two-dimensional Boussinesq Navier-Stokes equations,

$$
\begin{gathered}
\frac{\partial \boldsymbol{u}^{*}}{\partial t}+\boldsymbol{u}^{*} \cdot \nabla \boldsymbol{u}^{*}= \\
\frac{\partial \rho^{*}}{\partial t}+\boldsymbol{u}^{*} \cdot \nabla \rho^{*}=\kappa \nabla^{2} \rho^{*} \\
\nabla \cdot \boldsymbol{u}^{*}=0 .
\end{gathered}
$$


Dimensional variables, denoted with a star, are: velocity, $\boldsymbol{u}^{*}\left(x^{*}, z^{*}, t^{*}\right)=\left(u^{*}, w^{*}\right)$, density, $\rho^{*}\left(x^{*}, z^{*}, t^{*}\right)$, a reference density $\rho_{o}^{*}$, pressure $p^{*}\left(x^{*}, z^{*}, t^{*}\right)$, the acceleration due to gravity, $g$, and the unit vector in the positive $z$-direction, $\hat{k}$. The viscosity is $v$, and $\kappa$ is the density diffusion coefficient. Subsequently, we use the non-dimensional (non-starred) velocity, pressure, density, length and time scales,

$$
\left.\begin{array}{l}
\boldsymbol{u}^{*}=\boldsymbol{u} \sqrt{g^{\prime} H}, p^{*}=p \rho_{o}^{*} g^{\prime} H, \rho^{*}=\rho \rho_{o}^{*}, x^{*}=x H, \\
z^{*}=z H, h^{*}=h H, t^{*}=t H / \sqrt{g^{\prime} H},
\end{array}\right\}
$$

where

$$
g^{\prime}=g\left(\rho_{b}-\rho_{t}\right) / \rho_{t}
$$

is the reduced gravity, based on the ambient density difference. Note that the dimensional ambient density profile is $\bar{\rho}^{*}=\bar{\rho} \rho_{o}^{*}$ and bottom and top densities are $\rho_{b}^{*}=\rho_{b} \rho_{o}^{*}$ and $\rho_{t}^{*}=\rho_{t} \rho_{o}^{*}$. The reduced gravity, based on the gravity current density anomaly, is

$$
g^{\prime} / S=g\left(\rho_{c}-\rho_{t}\right) / \rho_{t},
$$

where

$$
S=\frac{\rho_{b}-\rho_{t}}{\rho_{c}-\rho_{t}} \leqslant 1
$$

is a parameter that compares the ambient stratification to the gravity current density anomaly.

The normalized gravity current front speed is

$$
U=\frac{U^{*}}{\sqrt{g^{\prime} H}} .
$$

The stratification supports a full spectrum of internal waves with a first-mode longwave speed, $c_{o}^{*}=c_{o} \sqrt{g^{\prime} H}$, obtained from a linear eigenvalue problem in $\bar{\rho}(z)$ (Baines 1997). The ratio of the gravity-current front speed to the linear long-wave speed defines the Froude number,

$$
\operatorname{Fr}=U / c_{o} .
$$

For the case of linear stratification with constant $\mathrm{d} \bar{\rho} / \mathrm{d} z=-1, c_{o}=1 / \pi$, and $F r=\pi U$.

A second gravity-current scaling can be defined based on the density difference between the current and the ambient fluid,

$$
U_{g c}=\frac{U^{*}}{\sqrt{g^{\prime} H / S}}=U \sqrt{S}
$$

where subscript $g c$ denotes classic Benjamin gravity-current scaling. Note that the limit $S \rightarrow 0$ represents the classic gravity-current problem. However, here we require this limit to be achieved by reducing the ambient stratification, such that $\left(\rho_{b}-\right.$ $\left.\rho_{t}\right) \rightarrow 0$, rather than $\left(\rho_{c}-\rho_{t}\right) \rightarrow \infty$, since all theory we present makes the Boussinesq assumption, $\left(\rho_{c}-\rho_{t}\right) / \rho_{t} \ll 1$.

The front speed is frequently normalized in yet a third way using the reduced gravity based on the gravity current, $g^{\prime} / S$, and the gravity current height, $h$,

$$
U_{h}=\frac{U^{*}}{\sqrt{g^{\prime} h / S}} .
$$

We note that (2.9) is frequently refered to as the gravity current Froude-number condition (to be distinguished from (2.7)). Each of the three normalizations, $U, U_{g c}$ and $U_{h}$, reveal different gravity-current properties. 


\section{Internal solitary waves}

Large-amplitude internal waves frequently observed in the ocean, atmosphere and laboratory experiments are long and maintain coherence over long propagation distances. The canonical weakly nonlinear theory that captures these features is the Korteweg-de Vries (KdV) equation, which includes the competing effects of nonlinearity and non-hydrostatic dispersion. An extended KdV (eKdV) equation, which adds cubic nonlinearity (see Miles 1980) is frequently employed.

Fully nonlinear theories can be obtained from (2.1) by neglecting viscosity and diffusion, assuming a frame of reference moving with the wave speed, $c$, and integrating the momentum equations along a streamline originating in the undisturbed fluid (see figure 1). This yields the Dubreil-Jacotin-Long (DJL) equation (Lamb 2002) for the streamline displacement, $\eta(x, z)$ (measured relative to the ambient),

$$
\nabla^{2} \eta+\frac{N^{2}(z-\eta)}{c^{2}} \eta=0,
$$

with boundary conditions $\eta=0$ at $z=0,1$ and $\eta \rightarrow 0$ as $x \rightarrow \pm \infty$. Here, $N$ is the dimensionless Brunt-Väisälä frequency, $N^{2}=-\mathrm{d} \bar{\rho} / \mathrm{d} \bar{z}$. This is an eigenvalue problem for $c$ and $\eta(x, z)$ for a solitary wave centred at $x=0$. DJL solutions become broadcrested with increasing amplitude, and approach an infinite-wavelength solution that, in the half-plane, represents a special energy-conserving bore (Brown \& Christie 1998; Lamb \& Wan 1998), with uniform upstream and downstream regions connected by a (dissipationless) jump. In this limit, the flat isopycnals in the disturbed region are said to be conjugate to those in the undisturbed region (termed originally by Benjamin 1966), and we refer to this dissipationless bore solution as the conjugate state. The conjugate state is the solution, $\eta(z)$, to the one-dimensional eigenvalue problem

$$
\eta^{\prime \prime}(z)+\frac{N^{2}(z-\eta)}{c^{2}} \eta=0,
$$

subject to the boundary conditions

$$
\eta(0)=\eta(1)=0 .
$$

For certain density profiles, solutions to (3.1) do not reach the broad-crested limit as the wave amplitude is increased, but rather develop a convective instability marked by a recirculating core of trapped fluid. The onset of instability, as amplitude is increased, occurs when $u(0)=c$ (for waves of elevation), a condition termed the breaking limit (Lamb \& Wan 1998). Solutions beyond the breaking limit were computed by Brown $\&$ Christie (1998), but because the densities in the core are outside the range of the ambient density and exhibit gravitationally unstable stratifications in the core, solutions are unphysical. Derzho \& Grimshaw (1997) found weakly nonlinear solitarywave solutions with trapped cores in near-linear stratification by matching an outer region where streamlines are continuous and satisfy (3.1), with a core region of constant density and uniform vorticity. These waves become broad-crested with increasing amplitude, approaching a bore-like solution similar to the conjugate state, but with a trapped core. While conjugate states without trapped cores represent a dissipationless bore, conjugate states with trapped cores can be thought of as special energy-conserving gravity currents, with height $h_{C S}$. Lamb \& Wilkie (2004) found conjugate states (3.2) with trapped cores for arbitrary ambient stratification. The boundary conditions of (3.2) are modified to $\eta\left(h_{C S}\right)=h_{C S}, \eta(1)=0$, where $h_{C S}$ denotes the conjugate state trapped core (energy-conserving gravity current) height. The external solution is matched to the core by applying Bernoulli's equation along 
the separating streamline and a momentum balance over the full depth. The theory is limited to situations where the core density is equal to the bottom density (implying $S=1$ ) and the vorticity in the core is arbitrary. We will extend the theory to calculate gravity currents with arbitrary $S$, but take the core vorticity to be zero.

The nonlinear internal-wave solutions are also closely related to upstream wave generation in stratified flow over topography. The DJL equation (3.1) is identical to Long's model (Baines 1997) for a steady flow approaching a bottom topographic disturbance. For certain subcritical $(F r<1)$ conditions, Long's model predicts upstream-propagating disturbances that violate the assumption of steady uniform approach flow. These degenerate solutions are identical to the two-dimensional wavebreaking DJL solutions. Moreover, in a frame moving at speed $c$, a trapped core may be thought of as a topographic bump and the upstream ambient fluid as the uniform approach flow. Ungarish (2006) used this approach along with a one-dimensional Long's model to compute steady solutions for gravity currents in a linearly stratified fluid. He recognized that solutions become unphysical at the breaking limit, signifying the onset of upstream-propagating disturbances, but as in Long's model, all $\mathrm{Fr}>1$ solutions are valid. However, the connection between the breaking and internal wave generation was not fully explored.

The dam-break solutions shown in figure 2 show the connections between gravity currents, Long's model, and conjugate states with trapped cores. For small dam height, $F r<1$ and the gravity-current fluid generates waves consistent with upstream propagation in Long's model (figure $2 a$ ). However, for large dam height, $\mathrm{Fr} \gtrsim 1$ and a pure gravity current is obtained (figure $2 b$ ) which is consistent with the trapped core conjugate state. The smooth streamline displacement over the gravity current is also consistent with supercritical Long's model solutions. In general, smaller dam heights are more likely to generate waves, and large heights, steady gravity-current solutions, but the crossover will depend on the ambient stratification and the parameter $S$.

Here, we extend the conjugate-state wave theory of Lamb \& Wilkie (2004) to compute steady gravity-current solutions for arbitrary core density and ambient stratification. It will be shown that the trapped-core conjugate-state solutions are analogous to Benjamin's energy-conserving gravity-current solution, and that the two are equivalent in the limit of small $S$. Moreover, by allowing dissipation, the Lamb \& Wilkie theory can yield solutions for a range of $h$. These dissipative gravity currents can be calculated for arbitrary stratification, and overlap with the results of Ungarish (2006) for linear $\bar{\rho}(z)$. However, steady solutions are limited by the onset of convective instability when $u\left(h_{C S}\right)=C$. From full numerical dam-break solutions, it is shown that this limit marks a transition between steady gravity currents and those that generate internal solitary waves in the fluid ahead.

\section{Steady gravity current theory}

Here a theory for steady gravity currents is developed by adapting the trapped-core conjugate-state theory of Lamb \& Wilkie (2004). The streamline displacement, $\eta(z)$, is computed by assuming the gravity current is long, and the flow above the gravity current is approximately uniform in $x$, enabling the hydrostatic approximation. As a result, the region near the nose, where the flow changes rapidly, is not resolved. However, evidence that the isopycnal displacement through the front of a trapped core is smooth and resembles a gravity-current front is provided in the two-dimensional trapped core solutions of Derzho \& Grimshaw (1997). The theory will yield steady solutions for a range of $h$ and $\bar{\rho}(z)$. Gravity currents with heights different from the 
conjugate state, $h \neq h_{C S}$, can be found, but these solutions implicitly require energy dissipation. The mechanism(s) for this dissipation are not addressed, but the theory presented makes an implicit assumption regarding the form, namely that it is uniform over depth.

First, consider a gravity current whose front moves with steady speed, $U$. In a frame of reference moving with the current, the variables in the upstream ambient region (see figure 1), denoted with an overbar, are

$$
(\bar{u}(\bar{z}), \bar{\rho}(\bar{z}), \bar{p}(\bar{z}))=\left(-U, \bar{\rho}(\bar{z}), \bar{p}(0)-\int_{0}^{\bar{z}} \frac{\bar{\rho}\left(\bar{z}^{\prime}\right)}{\rho_{b}-\rho_{t}} \mathrm{~d} \bar{z}^{\prime}\right) .
$$

Here, $\bar{z}=z-\eta(z)$ denotes the upstream vertical coordinate, whereas $z$ is defined as positive from the bed in the region through the current. The pressure is hydrostatic and we take the reference density $\rho_{o}=\rho_{t}$. From continuity between isopycnals, $u \mathrm{~d} z=-U \mathrm{~d}(z-\eta)$, so that the velocity (in the moving frame) above the gravity current is

$$
u=U\left(\frac{\mathrm{d} \eta}{\mathrm{d} z}-1\right), \quad z \geqslant h
$$

and inside the gravity current is

$$
u=0, \quad z<h .
$$

The stagnation pressure at the gravity current nose, by applying the Bernoulli equation along the bottom streamline originating in the upstream ambient, is $p_{s}=\bar{p}(0)+\left(\rho_{b} / \rho_{t}\right) U^{2} / 2$. Following this streamline back, the hydrostatic pressure within the current is

$$
p(z)=\bar{p}(0)+\frac{1}{2} \frac{\rho_{b}}{\rho_{t}} U^{2}-\frac{\rho_{c} z}{\rho_{b}-\rho_{t}}, \quad z<h,
$$

while above the current

$$
p(z)=\bar{p}(0)+\frac{1}{2} \frac{\rho_{b}}{\rho_{t}} U^{2}-\frac{\rho_{c} h}{\rho_{b}-\rho_{t}}-\int_{h}^{z} \frac{\bar{\rho}(z-\eta)}{\rho_{b}-\rho_{t}} \mathrm{~d} z, \quad z \geqslant h .
$$

The streamline displacement, $\eta(z)$, is given by the one-dimensional DJL equation (3.2), with the boundary conditions, $\eta(h)=h$ and $\eta(H)=0$. The DJL equation carries an implicit assumption that the Bernoulli energy dissipation is identical along all streamlines (independent of $z$ ). It will be shown that for the conjugate state, $h=h_{C S}$, the dissipation is everywhere equal to zero; otherwise dissipation is finite but uniform. No assumptions are made regarding the form or mechanism behind these losses.

Next, $U$ is determined by a flow-force balance. The momentum flux through a vertical section including the current and the fluid above it must balance the flux through a section in the upstream ambient fluid,

$$
\int_{0}^{H}\left[p(z)+u^{2}(z)\right] \mathrm{d} z-\int_{0}^{H}\left[\bar{p}(\bar{z})+\bar{u}^{2}\right] \mathrm{d} \bar{z}=0 .
$$

Substituting (4.1)-(4.5) above yields,

$$
\begin{aligned}
\int_{h}^{H}\left[p(z)-\bar{p}(z-\eta)\left(1-\eta^{\prime}\right)-\right. & \left.U^{2} \eta^{\prime}\left(1-\eta^{\prime}\right)\right] \mathrm{d} z \\
& +\int_{0}^{h}\left[\bar{p}(0)+\frac{1}{2} \frac{\rho_{b}}{\rho_{t}} U^{2}-\frac{\rho_{c}}{\rho_{b}-\rho_{t}} z\right] \mathrm{d} z=0 .
\end{aligned}
$$


Note that $\eta^{\prime}=\mathrm{d} \eta / \mathrm{d} z$ and in the upstream ambient the change of variable $\bar{z}=z-\eta(z)$, $\mathrm{d} \bar{z}=\left(1-\eta^{\prime}\right) \mathrm{d} z$ is used to simplify the second integral in (4.6), resulting in a change of integration limits after using $\eta(0)=h$. Equation (4.7) can be simplified by substituting the hydrostatic pressure relations (4.1) and (4.5) and integrating several times by parts using (3.2) and the boundary conditions, $\eta(h)=h$ and $\eta(H)=0$ (see details in the Appendix).

After the simplifications, the flow-force balance becomes

$$
\int_{h}^{1} \eta^{\prime}(z)^{3} \mathrm{~d} z-\frac{1-S}{S} \frac{h(2-h)}{\frac{1}{2} U^{2}}+\eta^{\prime}(h)^{2}(2-h)-2 \eta^{\prime}(h)(2-h)+2=0 .
$$

Overall, there are three unknowns: the gravity current height, $h$, and speed, $U$, and the pycnocline displacement, $\eta(z)$. There are two equations, the DJL equation (3.2), and the flow-force balance (4.8). Thus, it is not possible to determine both $U$ and $h$, except in the energy-conserving case $h=h_{C S}$ (see below), so we approach the problem by specifying $h$ and determining $U$. This is the same approach as used by Benjamin (1968) in his original analysis without statification and by Ungarish (2006) in linear stratification.

\subsection{Comparison with Benjamin's solution}

In the limit $S \rightarrow 0$, the ambient stratification is negligible and the gravity current dynamics should approach the classic solution of Benjamin (1968). Ungarish (2006) has already demonstrated this limit for the case of linear stratification (we will later show a more general comparison with Ungarish's results). For our general stratification theory, it can be verified that for $S \rightarrow 0$, the DJL equation (3.1) reduces to $\eta_{B}^{\prime \prime}=0$, with the solution $\eta_{B}=h(z-1) /(h-1)$, where the $B$ subscript denotes Benjamin's solution. Substitution of $\eta_{B}$ into the flow-force balance (4.8) yields the current speed,

$$
U_{g c}=U \sqrt{S}=\sqrt{\frac{2 h(1-h / 2)(1-h)}{1+h}},
$$

which is identical to Benjamin's solution.

\subsection{Energy dissipation}

The theory of Benjamin predicts that gravity currents are dissipative except in the special case $h=0.5$. Although dissipation is implied, the theory does not account for its source. An energy balance in terms of Bernoulli head along any streamline outside the gravity current can be written

$$
p(z)+\frac{1}{2} \bar{\rho}(z-\eta) u(z)^{2}+\frac{\bar{\rho}(z-\eta)}{\rho_{b}-\rho_{t}} \eta(z)+\Delta=\bar{p}(z-\eta)+\frac{1}{2} \bar{\rho}(z-\eta) U^{2},
$$

where $\Delta=\Delta^{*} /\left(\rho_{t} g^{\prime} H\right)$ is the head loss. By substituting the pressure and velocity expressions (4.1), (4.2) and (4.5), and using the expression for $p-\bar{p}(z-\eta)$ from (A 2), it can be shown that the head loss is equal to

$$
\Delta=\left(\frac{1-S}{S}\right)^{h}-\frac{1}{2} U^{2}\left[\eta^{\prime}(h)^{2}-2 \eta^{\prime}(h)+1\right] .
$$

Note in cases for which $\Delta>0$, energy dissipation is implicit in the gravity current solution. Moreover, dissipation is constant over depth, an implicit assumption of the DJL equation (3.2). Although we do not address mechanisms behind this dissipation, we do note that many of the steady solutions we find contain regions in which the 
Richardson number, $R i=N^{2} /(\mathrm{d} u / \mathrm{d} z)^{2}$, becomes critical $(R i \leqslant 0.25)$. These regions imply shear instability that can lead to mixing and are consistent with KelvinHelmholtz instabilities observed in the numerical calculations described later.

The flow force balance (4.8) can be decomposed into inviscid and head loss components,

$$
\int_{h}^{1} \eta^{\prime}(z)^{3} \mathrm{~d} z+h-\frac{(2-h)}{\frac{1}{2} U^{2}} \Delta=0,
$$

In the special case of $\Delta=0$, a third constraint, in addition to (3.1) and (4.8), is obtained for gravity-current solutions by setting (4.11) equal to zero. This constraint yields a value for $h$ corresponding to the energy-conserving gravity current for a given $S$ and $\bar{\rho}(z)$. When $S=1$, this solution is identical to the conjugate-state solution $\left(h_{C S}, U_{C S}\right)$ given by Lamb \& Wilkie (2004) for a trapped core with zero vorticity.

In the limit of $S \rightarrow 0$, the Benjamin limit, the head loss can be calculated from (4.11) and the solution $\eta_{B}=h(z-1) /(h-1)$, and is equal to

$$
\Delta_{B}=\frac{1-S}{S}\left[\frac{h^{2}(1-2 h)}{2\left(1-h^{2}\right)}\right],
$$

in agreement with Benjamin's original solution (this agreement was also shown for linear stratification by Ungarish 2006). Thus, as $S \rightarrow 0$, the conjugate-state internal wave with a trapped core is equivalent to a dissipationless gravity current with $h=0.5$.

\subsection{Computational method}

The system (3.2) and (4.8) must be solved numerically because of the nonlinearity of the DJL equation for arbitrary stratification. Following Lamb \& Wilkie (2004), the boundary-value problem (3.2) is solved using a shooting method that satisfies $\eta(h)=h$ and $\eta(1)=0$, and $U$ is determined using a Newton iteration that satisfies (4.8). If the dissipationless conjugate-state solution is sought, the additional condition, $\Delta=0$ from (4.11) is also satisfied with a second Newton iteration to determine $h$.

The additional constraint

$$
\frac{\mathrm{d} \eta}{\mathrm{d} z} \leqslant 1
$$

which from (4.2) is equivalent to requiring $u(z) \geqslant 0$, ensures that convective overturning does not occur in the domain, i.e. all streamlines originate in the upstream ambient fluid. Combinations of $\bar{\rho}(z)$ and $h$ which do not satisfy this criterion imply upstream propagating disturbances, and are rejected since this violates the steady-state assumption. The violation occurs when $u(h)=U$ and, in general, this condition is reached by steadily decreasing $h$ for a constant value of $S$.

For some combinations of $(S, h, \lambda)$, higher-mode solutions to the DJL equation (3.2) can be found. These higher modes have been described by Ungarish (2006) for constant $N$ stratification. We will primarily focus on the first-mode solutions, but in $\S 6.4$, we discuss higher modes in relation to the dam-break problem.

Internal wave modes in the region $[h, 1]$ above the gravity current depend on $u(z)$ and $\rho(z)$, and are given by the Taylor-Goldstein (T-G) equation (Baines 1997) after treating the top of the gravity current as a solid horizontal boundary. For long waves,

$$
\left(u-c_{j}\right) \frac{\mathrm{d}^{2} \phi_{j}}{\mathrm{~d} z^{2}}+\left[\frac{\mathrm{d} \rho / \mathrm{d} z}{u-c_{j}}-\frac{\mathrm{d}^{2} u}{\mathrm{~d} z^{2}}\right] \phi_{j}=0, \quad \phi_{j}(h)=\phi_{j}(1)=0,
$$

where the eigenvalues $c_{j}$ are the long-wave speeds and $\phi_{j}$ the corresponding eigenmodes. The largest neutral $\left(\operatorname{Im}\left(c_{j}\right)=0\right)$ eigenvalue is the fastest internal wave 


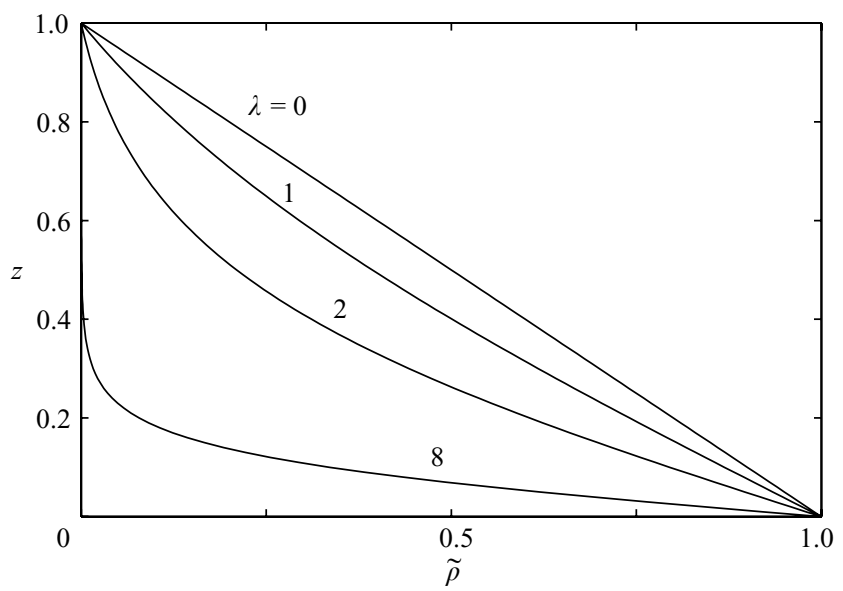

FIGURE 3. Density profiles (5.2) for several values of $\lambda$.

that can propagate toward the gravity current front, and we denote its speed $c_{l}$. The T-G neutral modes lie outside the range of the velocity profile, and thus the fastest mode exceeds the maximum velocity, $c_{l} \geqslant u(h)$. As $h$ is decreased for a given stratification, $c_{l}$ and $u(h)$ both approach $U$ from the left, that is $u(h) \leqslant c_{l} \leqslant U$. At a critical height and speed, $\left(h_{c}, U_{c}\right), c_{l}=u(h)=U$, implying that long waves propagate upstream and signifying incipient overturning. Beyond this point, solutions are unphysical owing to a violation of the assumption of steady upstream flow. The critical points depend on the stratification, $h_{c}(S, \bar{\rho}(\bar{z})), U_{c}(S, \bar{\rho}(\bar{z}))$, and define a lower boundary curve separating steady and unsteady gravity-current solutions.

An upper bound on steady solutions is given by the energy-conserving conjugatestate solution. Above the conjugate state, dissipation is negative, $\Delta<0$ for $h>h_{C S}$, an unphysical result in the absence of external energy sources. Thus for each $S, \bar{\rho}(z)$ combination, only solutions in the range $\left[h_{c}, h_{C S}\right]$ are accepted. Note that in the limit $S \rightarrow 1, h_{c}=h_{C S}$, since, from the head loss expression (4.11) the gravity current is dissipationless for $\eta^{\prime}(h)=1$ and $u(h)=U$.

\section{Steady gravity current solutions}

Here we show the results of the steady gravity current propagation theory. We study stable density profiles chosen from a family of hyperbolic tangent curves given by

$$
\bar{\rho}(z)=\rho_{t}(1+\Delta \rho \widetilde{\rho}(z)),
$$

where $\Delta \rho=\left(\rho_{b}-\rho_{t}\right) / \rho_{t}$ and

$$
\widetilde{\rho}=1-\frac{\tanh (\lambda z)}{\tanh \lambda} .
$$

These profiles have non-zero buoyancy frequency at $z=0$, and thus the conjugatestate solutions develop trapped cores (Lamb \& Wan 1998). The only exception is for $S=1$ and linear stratification which does not have a conjugate-state solution with a trapped core. The parameter $\lambda$ is a measure of the non-uniformity of the stratification. In the limit $\lambda \rightarrow 0, \widetilde{\rho}=1-z$ and the stratification is linear (constant $N$ ), whereas for $\lambda \gg 1$, the stratification is strongly bottom-trapped (figure 3 ).

Steady gravity-current solutions ( $U$ vs. $h$ ) are shown for a case $\lambda=8$ and $S=0.952$ in figure 4. Physically relevant steady solutions lie between the limits marked by the 


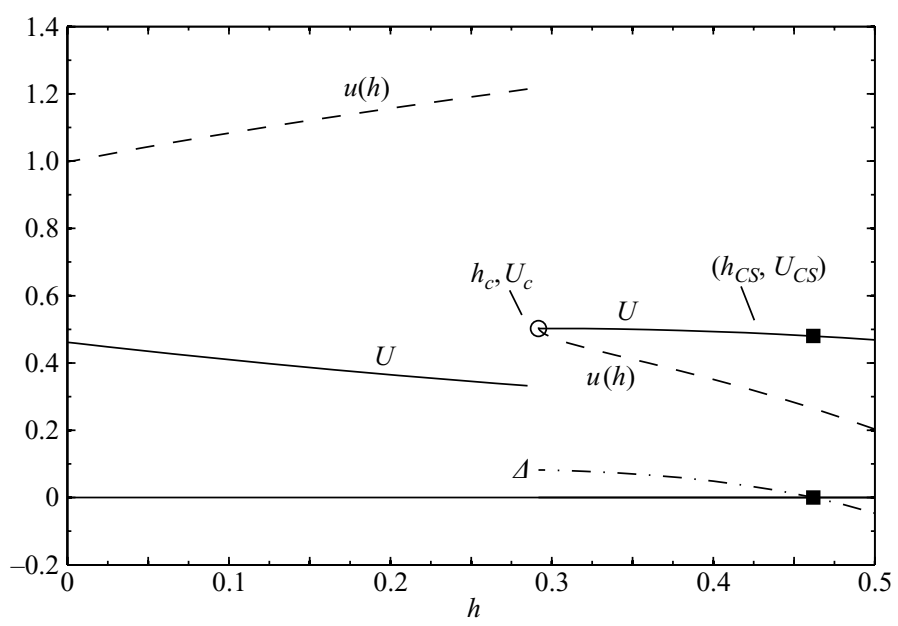

FIGURE 4. Gravity-current solutions for a fixed stratification and core density $(\lambda=8, S=0.952)$. Front speed, $U$, head loss, $\Delta$, and ambient fluid velocity directly above the current, $u(h)$, are shown as a function of gravity-current height, $h$. The conjugate-state solution $(\Delta=0)$ and the critical point for incipient overturning $(u(h)=U)$ are marked. Solutions on the lower branch, $h<h_{c}$, are unphysical because they are convectively unstable.
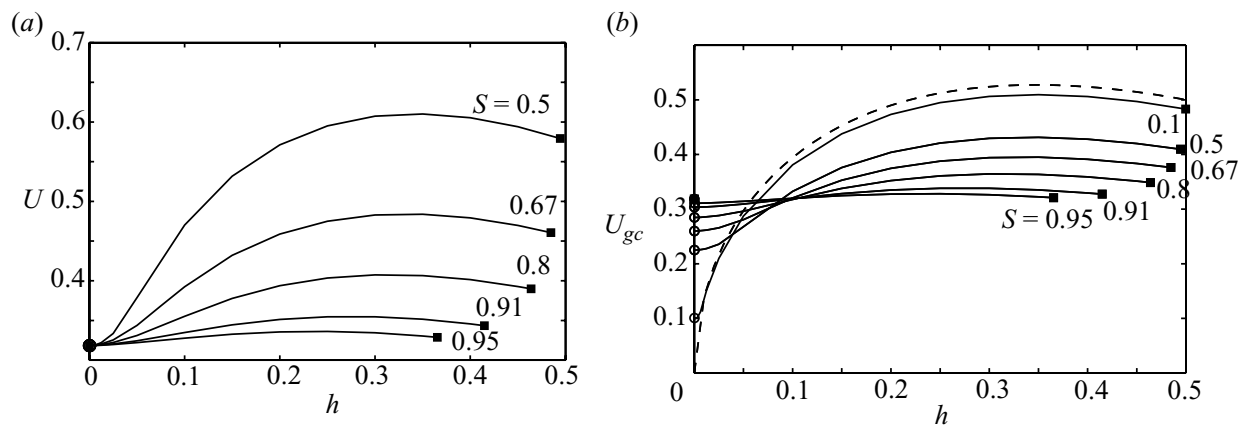

FIGURE 5. Gravity-current speed vs. height for curves for $\lambda=0$. Speed is normalized as $U$ in $(a)$ and $U_{g c}$ in (b). Conjugate-state solutions (squares); critical points (open circles). The $S=1$ case has only a single solution at $h=0, U=U_{g c}=1 / \pi$. The Benjamin curve from (4.9) (dashed line) is shown in $(b)$.

conjugate-state solutions $\left(h_{c s}, U_{c s}\right)$ and the critical point at which overturning begins $\left(h_{c}, U_{c}\right)$. The latter limit is the point at which $u(h)=U$ (see convergence of $u(h)$ and $U$ curves). Below the critical point, a second branch of solutions exists for which $u(h)>U$, but these violate the steady assumptions and are rejected. The head loss is also shown, with $\Delta=0$ for the conjugate-state solution, and invalid solutions with $\Delta<0$ for $h>h_{C S}$.

The (physical) $U v s . h$ solutions are shown for varying values of the density anomaly $S$ and for linear stratification $(\lambda=0)$ in figure 5 . Gravity-current speed is normalized as $U$ in figure $5(a)$, and $U_{g c}$ in figure 5(b), the latter for comparison with the Benjamin solution. In figure $5(a)$, the critical velocity is $U_{c}=1 / \pi$, independent of $S$, and equal to the linear long-wave speed, $c_{o}$. For $S=1, h_{C S}=0$, implying that there are no finiteheight energy-conserving gravity currents for the constant $N$ case. As $S$ decreases, both $h_{C S}$ and $U_{C S}$ increase. In figure $5(b)$, steady solutions lie below the Benjamin curve for large $h$, but exceed it for small $h$. The small $h$ and $S \approx 1$ solutions (with 
(a)

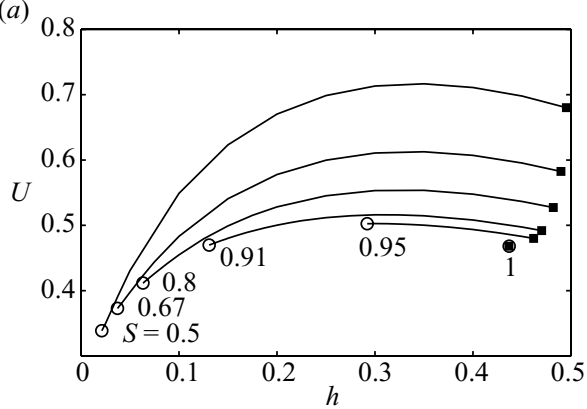

(b)

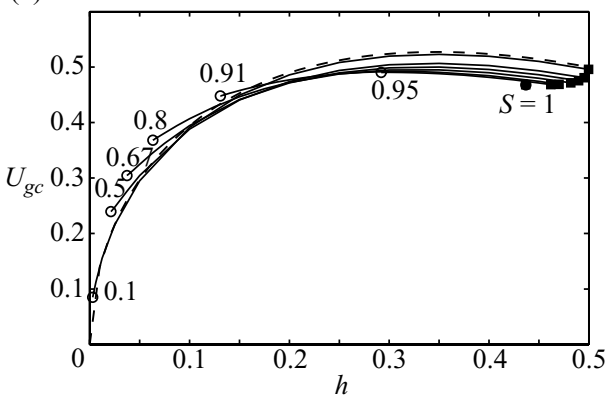

FIGURE 6. Gravity-current speed $v$. height for $\lambda=8:(a) U$ and $(b) U_{g c}$. Symbols, lines and $S$ values as in figure 5 .

(a)

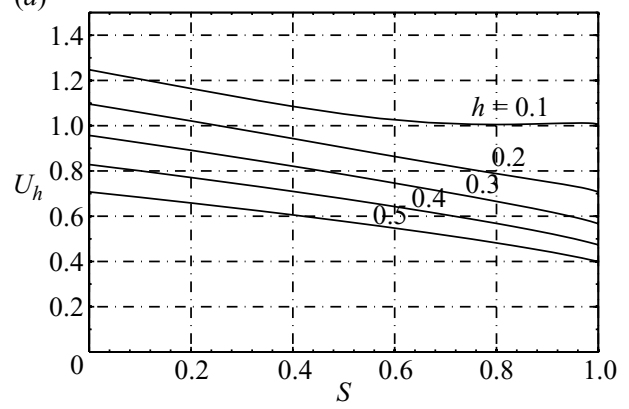

(b)

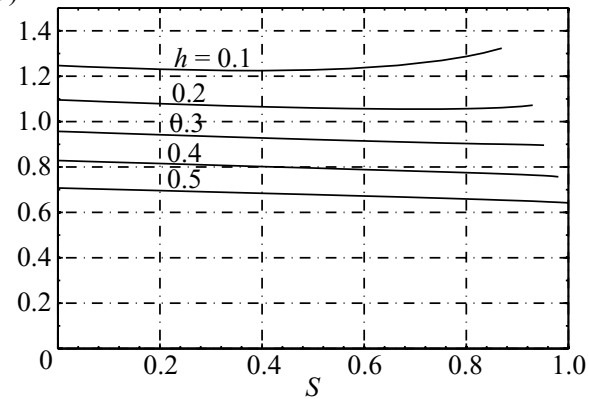

FIGURE 7. Gravity-current speed, normalized as $U_{h}$ (2.9), as a function of the core density ratio, $S$ for various gravity current heights. $(a)$ Uniform stratification $(\lambda=0)$. $(b)$ Non-uniform stratification $(\lambda=8)$.

$U \approx c_{o}$ ) are closer to the long-wave speed than the Benjamin gravity-current speed, but solutions approach the Benjamin curve as $S \rightarrow 0$. The conjugate-state height and speed increase with decreasing $S$, approaching the energy-conserving half-depth Benjamin solution $\left(h=0.5, U_{g c}=0.5\right)$ as $S \rightarrow 0$. For $\lambda=8$ (figure 6$), U_{c}$ increases with $S$, and $U_{g c}$ curves for all values of $S$ are approximated well by the Benjamin solution, provided that $h>h_{c}$.

In figure 7, the speed is normalized as $U_{h}(2.9)$. For $\lambda=0, U_{h}$ agrees with figure 3 of Ungarish (2006). For $\lambda=8, U_{h}$ is nearly independent of $S$ for a given $h$, and approaches the Benjamin solution for $h=0.5$ and $S \rightarrow 0$. However, steady solutions exist only for regions of $(h, U)$ above the critical overturning point, and thus the solution curves terminate as $S \rightarrow 1$.

In figure 8 , the critical points for convective overturning $U_{c}$ are shown as a function of gravity-current height, $h$, and normalized by $c_{o}$. These curves are generated by increasing $S$ (moving to the right along the curve). As $\lambda \rightarrow 0, U_{c} / c_{o} \rightarrow 1$, but $h_{c} \rightarrow 0$. No solutions produce overturning for $\lambda=0$, but for all $\lambda>0$, the wave-breaking limit is reached for combinations of $(h, F r)$ that lie below the corresponding curve.

The conjugate-state gravity-current speeds, $U_{C S} \sqrt{S}$, are shown in figure 9. For each $\lambda$, decreasing $S$ (increasing core density) increases the height and speed of the conjugate state. As $S \rightarrow 0$, Benjamin's dissipationless gravity-current solution $\left(U_{g c}=h=0.5\right)$ is approached. For constant $N$, there is a notably sharp kink in the behaviour of $U_{C S}$. For $S=1, U_{C S}$ is equal to the linear wave speed, $1 / \pi$, whereas 


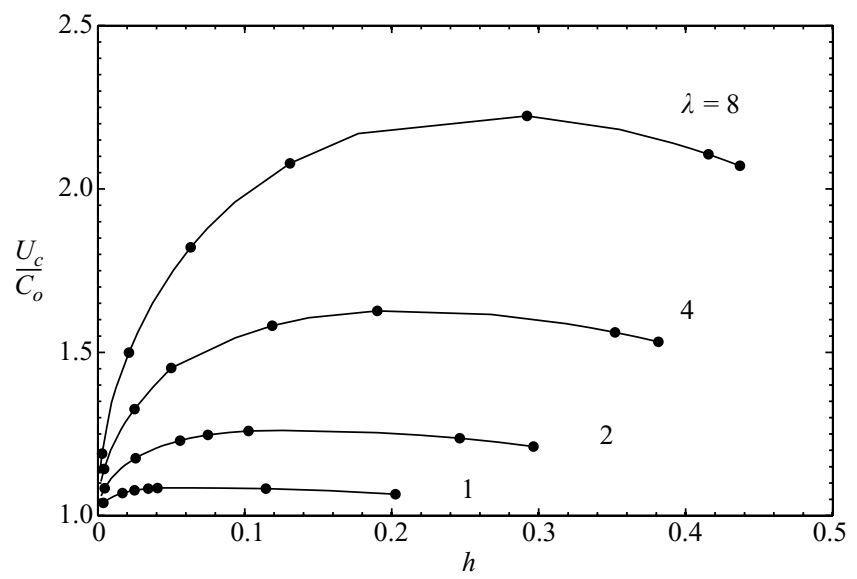

FIGURE 8. Critical gravity-current speed, normalized as $U_{c} / c_{o}$. Curves are generated by increasing $S$ from $S=0$ (pure gravity current) to $S=1$; dots (moving from right to left along a curve) represent $S=[1,0.99,0.95,0.91,0.8,0.5,0.1]$. For a given $\lambda$, no solutions can be found below the critical curve. Note that for $\lambda=0, U_{c} / c_{o}=1$ for all $S$ (also see figure 5).

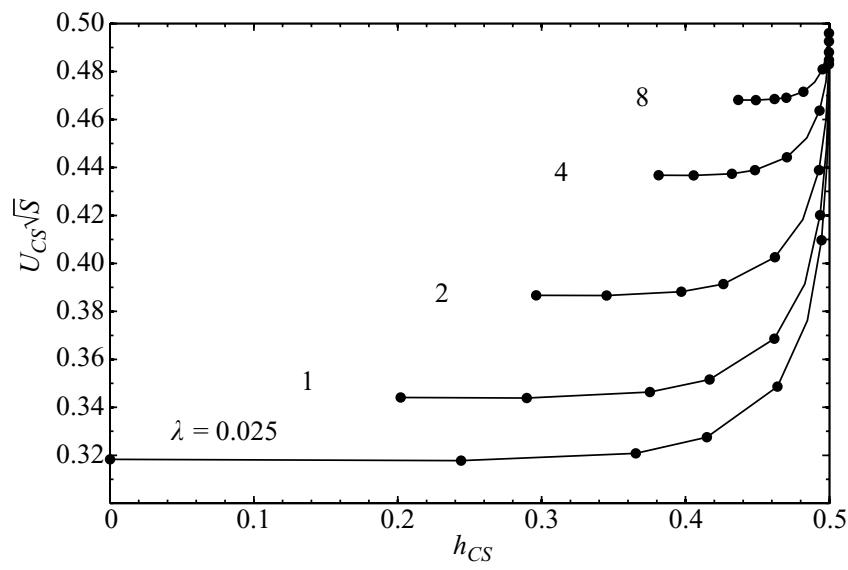

FIGURE 9. Conjugate-state speed, $U_{C S} \sqrt{S}=U_{g c}$, vs. height, $h_{C S}$, for varying $\lambda$. For each $\lambda$, curves are generated by decreasing $S$ from $S=1$ (intrusion) to $S=0$ (pure gravity current); dots (moving from left to right along a curve) represent $S=[1,0.99,0.95,0.91,0.8,0.5,0.1]$. Each curve tends to the energy-conserving Benjamin solution, $h=0.5, U_{g c}=0.5$, as $S \rightarrow 0$.

for $S=0, U_{C S}$ is the Benjamin gravity-current speed. Non-uniform $N$ cases are much faster than the linear wave speed for $S=1$, starting out closer to the Benjamin limit (e.g. $\lambda=8$ ). In the limit of either $\lambda \rightarrow \infty$ for any $S$, or $S \rightarrow 0$ for any $\lambda$, the conjugate state is identical to the energy-conserving Benjamin solution.

\section{Numerical dam-break calculations}

\subsection{Numerical details}

To provide a comparison with the steady-gravity-current theory and to investigate the evolution of the full initial-value problem, simulations of gravity currents and intrusions were carried out using a non-hydrostatic two-dimensional Navier-Stokes solver. The numerical model uses a second-order projection method based on that 
of Bell \& Marcus (1992). The method uses a Godunov-type evaluation of the nonlinear terms (a non-oscillatory finite-volume formulation) and is ideally suited to gravity-current flows where sharp gradients of density and velocity naturally develop. The governing equations (2.1) are solved with no explicit dissipation or diffusivity, $\nu=\kappa=0$. The numerical method does introduce numerical dissipation and mixing, but an important property of the advection scheme is that the numerical diffusion is significant only where large gradients (e.g. shocks) occur on the grid scale. This class of non-oscillatory finite-volume schemes are mass and momentum conserving and have dissipation properties comparable to large-eddy simulation (LES) models and have therefore been termed implicit-LES models (Margolin, Rider \& Grinstein 2006).

The numerical method has been used successfully for studies of large-amplitude internal solitary waves (Lamb 2002), and tests of the code show that large internal solitary waves can propagate distances of $\sim 100 \mathrm{H}$ with the correct phase speed, form, and minimal loss of energy $(<1 \%)$. As a further check of the model, $\lambda=0$ results were compared with the constant $N$ simulations in Maxworthy et al. (2002). Gravitycurrent propagation speeds, $U_{g c}$, and the density structure compared favourably, even though Maxworthy et al. used a no-slip bottom condition compared with our free-slip condition. A few calculations were carried out both with small uniform viscosity (giving a Reynolds number of $R e=\sqrt{g^{\prime} H} H / v=1 \times 10^{8}$ ), and with density and momentum diffusion calculated using a Smagorinsky-type closure (Afanasyev \& Peltier 1998). Very little dependence on the dissipation scheme was observed.

The ambient density structure, $\bar{\rho}(z)$ from (5.1), the gravity-current core density, $S^{-1}$, and the lock height, $h_{o}$, were each varied independently. Velocity, lengths, time and density were normalized as described previously. Calculations were carried out in a rectangular domain of unit height and dimensionless length ranging from 60 to 100, and with typical resolutions of 2000 in the horizontal and 200 in the vertical. Slip boundary conditions were applied at all boundaries. Each run was started from a dam-break initial condition, with a 'lock' of height $h_{o}$ and length $L_{o}$, and the initial density field given by

$$
\widetilde{\rho}_{i}(x, z)= \begin{cases}1 / S, & z \leqslant h_{o}, x \leqslant L_{o}, \\ \widetilde{\rho}(z), & z>h_{o}, x \leqslant L_{o}, \\ \widetilde{\rho}(z), & x>L_{o} .\end{cases}
$$

For $t>0$, disturbances are generated which propagate in both directions from the dam at $x=L_{o}$. Eventually, these disturbances will reflect from the lateral endwalls and reach the current front, affecting its propagation. The phase prior to the interaction between the current and the reflected waves, in which the current propagates with constant speed, is termed the slumping stage (Ungarish \& Huppert 2002). In our simulations, we focus entirely on this initial phase by making $L_{o}=20$ and the horizontal domain length equal to 100, sufficiently long to avoid the effects of endwall reflections. Both the domain and lock aspect ratios $(1: 100 ; 1: 20)$ are very small compared with most numerical and experimental studies (typically $1: 10 ; 1: 1$ ). However, they are appropriate for some natural conditions, such as river plumes, which can have vertical to horizontal extents of tens of metres to several kilometres. An estimate of the gravity-current height, $h$, during propagation is made for each numerical case by taking the first local maximum of the $\rho=\left(\rho_{c}+\rho_{b}\right) / 2$ contour in the direction moving from the undisturbed upstream region towards the gravity current. The height of this maximum is averaged over discrete times $t \leqslant 30$ following the initial release to produce a mean $h$ used in the analysis. 
Each set of numerical calculations was carried out holding fixed $\bar{\rho}(z)$ and $S$, and varying $h_{o}$, in order to examine the effect of increasing dam height on current propagation and wave generation. Because the dam height is a measure of the available potential energy, the relative comparison between the initial energy in the dam and the energy required by gravity-current solutions, particularly the conjugate state, has implications for steady $v$ s. wave-generating solutions.

\subsection{Numerical results}

A representative numerical solution is shown in figure 10 for the case $\lambda=0, S=0.91$, $h_{o}=0.5$. For this case $F r=0.93$, indicating a gravity current which is subcritical with respect to the linear long-wave speed. The fluid within the gravity current core, defined by the region in which $\rho \geqslant\left(\rho_{c}+\rho_{b}\right) / 2$, is indicated by the grey shading. Waves are generated at the gravity current front which detach, outrun and carry fluid from the front with them. These results are similar to the subcritical gravity currents of Maxworthy et al. (2002). The position of the front, $x_{f}$, is plotted with time in figure $10(\mathrm{~g})$ and compared to the position of the leading wave, calculated as the foremost point at which $\eta>0.001$ for the $\rho=0.5$ isopycnal. The front speed, $U_{g c}$, is calculated for each case by the best-fit line through the $x_{f} v$ s. $t$ curve (figure $10 g$ ). Comparison of the two slopes shows that the wave outruns the current. The point at which the wave first detaches, bringing core fluid with it, can be seen in the abrupt negative jump in the front position around $t=26$. The gravity-current speed, $U_{g c}$, is computed from the propagation prior to this time. The gravity-current speed is constant during this initial period, despite the presence of waves. Moreover, the gravity current does regain its initial speed $U_{g c}$ following the breakaway of core fluid, and the process repeats with the next wave, as observed by Maxworthy et al. (2002).

The waves generated by the gravity-current collapse are highly nonlinear (the maximum streamline displacement of the leading wave is approximately 0.2 for this simulation). A lower bound for the leading-wave speed is the linear long-wave speed $c_{o}$, but the actual speed is greater owing to the nonlinearity. It would appear that the greatest interaction between the current and the waves occurs when the nonlinear wave speed is close to the speed of the current.

As the height of the dam is increased for a given $\bar{\rho}(z)$ and $S$, the resulting currents undergo a series of transitions. These are shown in figure 11 for $\lambda=0$ and figure 12 for $\lambda=8$. For small $h_{o}$, the gravity current is slow and waves propagate upstream ahead of the gravity current. In figure 11(a), the flow above the gravity current head resembles a mode- 2 disturbance, with isopycnals displaced upward near the bottom, and downward at the top, but further upstream is a train of mode- 1 nonlinear waves in the form of an undular bore. As $h_{o}$ is further increased, finite-amplitude mode- 1 waves become apparent ahead of the gravity current (figures $11 b$ and 12a). These waves are initiated near the head of the current, but they quickly outrun it. The wave amplitude increases with increasing $h_{o}$, and they may carry a core of dense fluid from the current (see the leading wave in figure 11b), and there is also evidence of convective overturning inside the waves (figures $11 b$ and $12 a, b$ ), both characteristic of internal solitary waves with trapped cores (Lamb 2002). In some cases, further increase of $h_{o}$ results in a single large-amplitude wave that remains locked to the front of the gravity current over the course of the simulation (figure 12b). Although the waves do modify the current, at times resulting in an irregular rate of advance, the mean front speed is approximately constant over time, demonstrated in the figures by the good agreement between the data and the best linear fit to $x_{f} v s$. $t$ (figures $11 a, b$ and $12 a, b)$. This constant propagation speed is consistent with the 'slumping' phase, 
(a)

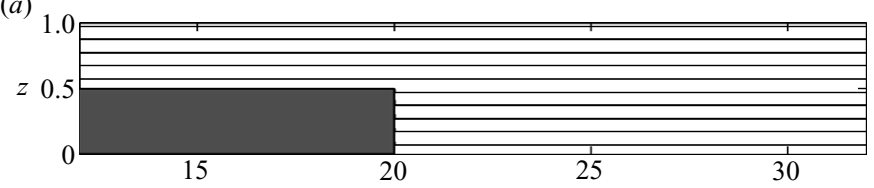

(b)

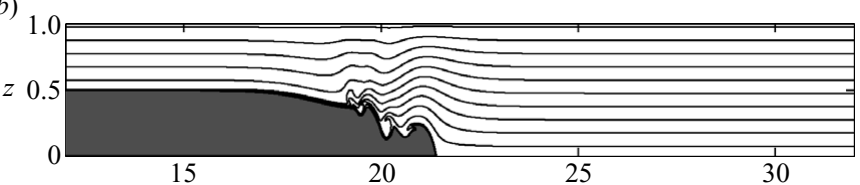

(c)

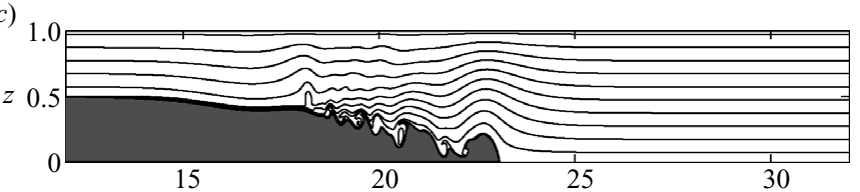

(d)

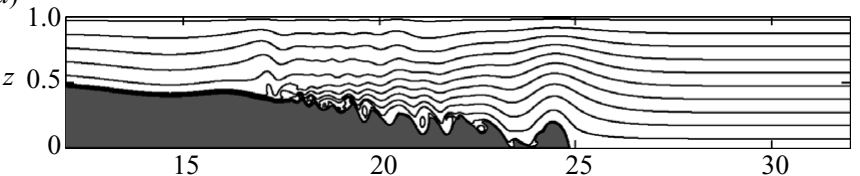

(e)

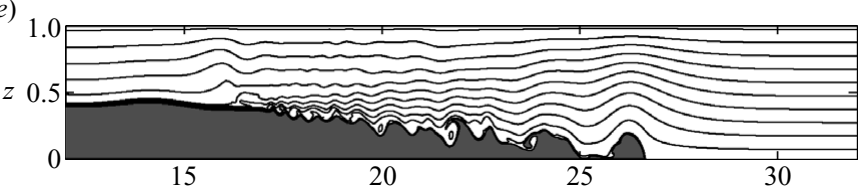

(f)

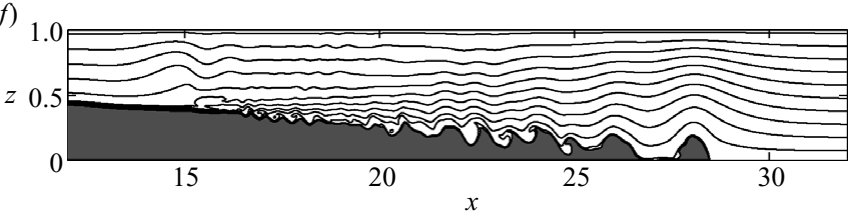

$(g)$

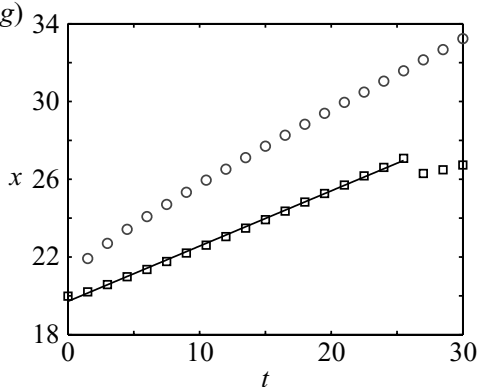

FIGURE 10. Representative gravity-current calculation beginning from a lock release $(\lambda=0$, $S=0.91$ ). Snapshots of the density contours are shown for dimensionless times $(a) t=0$, (b) $t=6,(c) t=12,(d) t=18,(e) t=24$ and $(f) t=30$. The gravity-current core, containing fluid that originated in the dam, is shown in solid grey. $(g)$ The position of the gravity current front (squares) and the leading-wave disturbance (circles) with time. The speed, $U_{g c}$ is computed from the best-fit line through the initial propagation prior to front regression. 
(a)
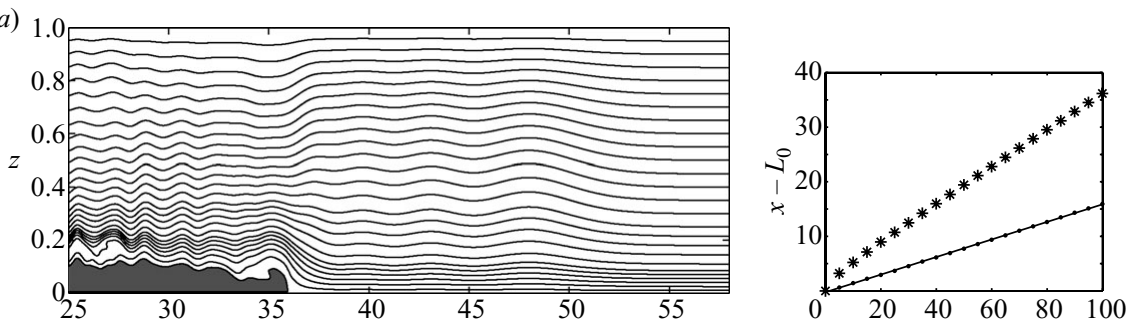

(b)
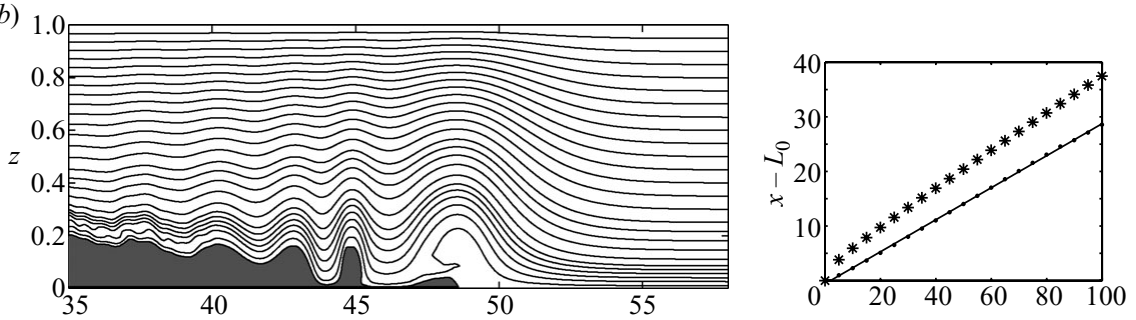

(c)
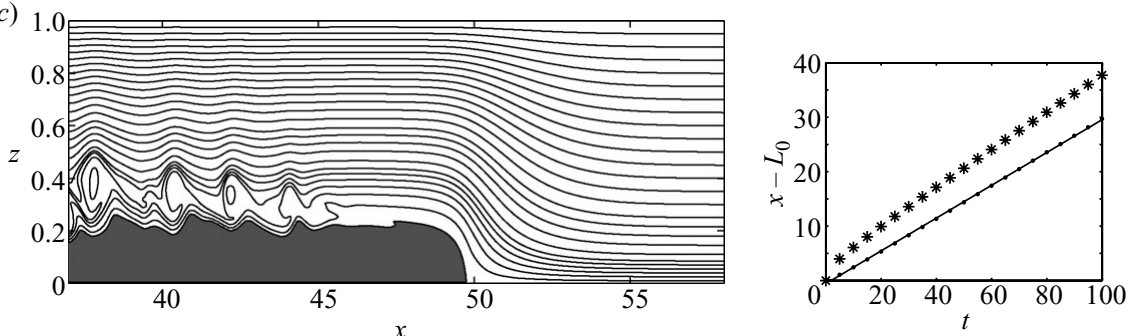

FIGURE 11. Gravity currents and upstream waves for $\lambda=0$ and $S=1$ (left-hand side). Gravity current $(\cdot)$ and leading-wave $(*)$ front positions $v$ s. time following lock release (right-hand side). (a) $h_{o}=0.4,(b) h_{o}=0.7,(c) h_{o}=0.9$. The gravity-current core is shown in grey, density contours are spaced more closely near the current for detail. The plots represent a transition from upstream-propagating internal waves $(a-b)$ to steady gravity-current flow $(c)$. The lock is at $x=20$.

identified in Ungarish \& Huppert (2002), which here extends over the duration of the simulation because the long lock negates end effects.

When $h_{o}$ is sufficiently large, upstream waves are not observed (figures $11 c$ and $12 c$ ). The current is supercritical with respect to internal waves, and the flow near the head is approximately steady in the frame moving with $U$, resembling a Benjamin gravity current with nearly constant thickness behind the front. Kelvin-Helmholtz billows are observed over the gravity current, but they are swept behind the current and do not affect the front region, though they contribute to mixing between the current and the ambient. These two-dimensional numerical simulations do not adequately resolve this mixing, and its description is beyond the scope of the paper. However, the Kelvin-Helmholtz instabilities are consistent with the critical Richardson number $(R i \leqslant 0.25)$ condition found in some of the steady gravity current solutions.

The steady, large $h_{o}$ cases can be interpreted as the trapped core conjugate state for $\bar{\rho}(z)$ with core density $\rho_{c}$. Consistent with this interpretation, the isopycnal structure around the gravity current in figures $11 c$ and $12 c$ resembles supercritical Long's model solutions with the gravity current playing the role of the topography. The smaller $h_{o}$ cases that generate waves in front of the gravity current illustrate the 
(a)
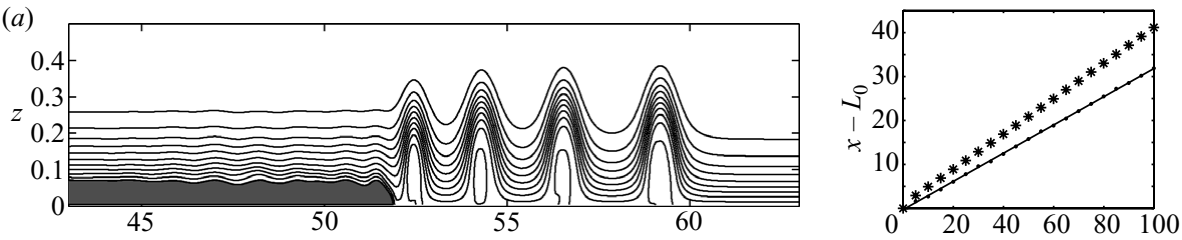

(b)
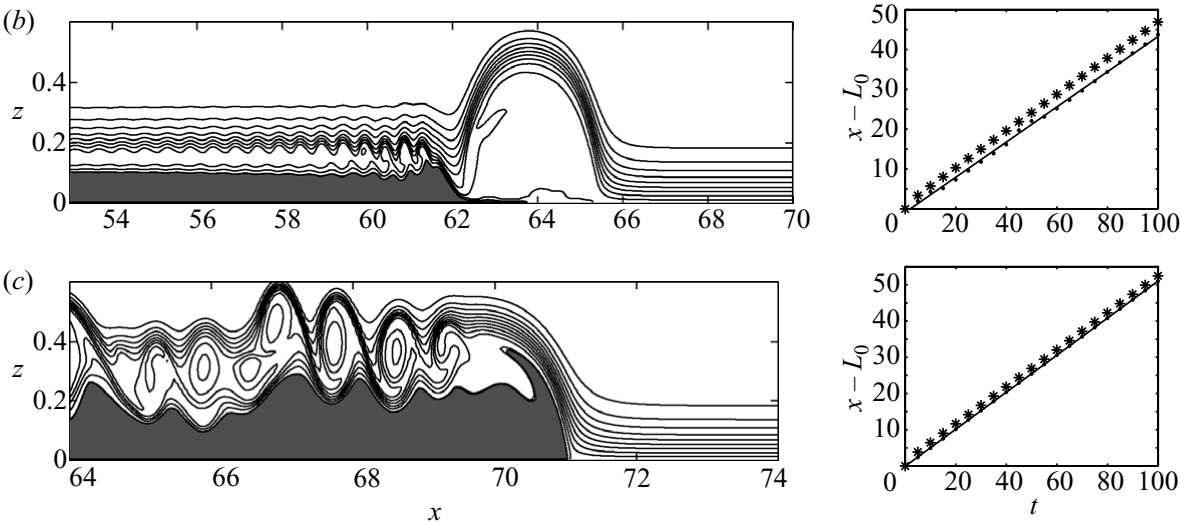

FIGURE 12. Gravity currents and upstream waves for $\lambda=8$ and $S=0.8$ (left-hand side). Gravity-current $(\cdot)$ and leading-wave $(*)$ front positions vs. time following lock release (right-hand side). (a) $h_{o}=0.25$, (b) $h_{o}=0.4$, (c) $h_{o}=0.7$. Transition through upstream-propagating internal waves $(a)$, a single trapped large-amplitude wave $(b)$ and a steady gravity current $(c)$. The lock is at $x=20$.

upstream propagation expected when $u(h)>U$. The finite-amplitude solitary-type waves observed in these cases are also consistent with resonant flow over topography explored by Grimshaw \& Smyth (1986), Melville \& Helfrich (1987), and Grimshaw \& Yi (1991), which have shown that within a range around critical flow, $F r=1$, steady solutions are not possible because nonlinear internal waves (solitary and undular bores) propagate into the undisturbed ambient flow. For uniform stratification, even very small topography can produce waves with a large-amplitude response owing to nonlinearity. The resonant band increases with the height of the topography (Grimshaw \& Smyth 1986). In the dam-break problem, $h$ and $U$ are not decoupled, as in fixed topography, yet the presence of the waves implies that this type of resonant forcing mechanism occurs near $F r=1$. In the next section, the limits of wave-generating and steady gravity-current solutions are delineated.

\subsection{Wave-gravity-current regime diagrams}

Based on the results of the steady theory $(\S \S 4-5)$, the numerical results can be grouped into gravity current and internal wave regimes. Steady solutions are predicted to lie between the overturning limit $\left(U=u(h)=c_{l}\right)$ and the conjugate state. In figures $13(a)(\lambda=0)$ and $13 b(\lambda=8)$, numerical results for $U_{g c}$ as a function of $S$ are plotted with the relevant curves from the steady theory: the upstream generation limit $U_{c}$, the conjugate-state speed, $U_{C S}$, and the linear long-wave speed for the ambient stratification, $c_{o}$, which defines critical $(F r=1)$ flow. The numerical results are separated into the three regimes identified above. Note that for a constant $S$, increasing $U_{g c}$ corresponds to increasing lock height, $h_{o}$.

For $\lambda=0$ (figure 13a), numerical solutions that generate internal waves lie entirely within the subcritical domain $\left(F r=U / c_{o}<1\right)$. The $F r=1$ boundary (lower dashed 

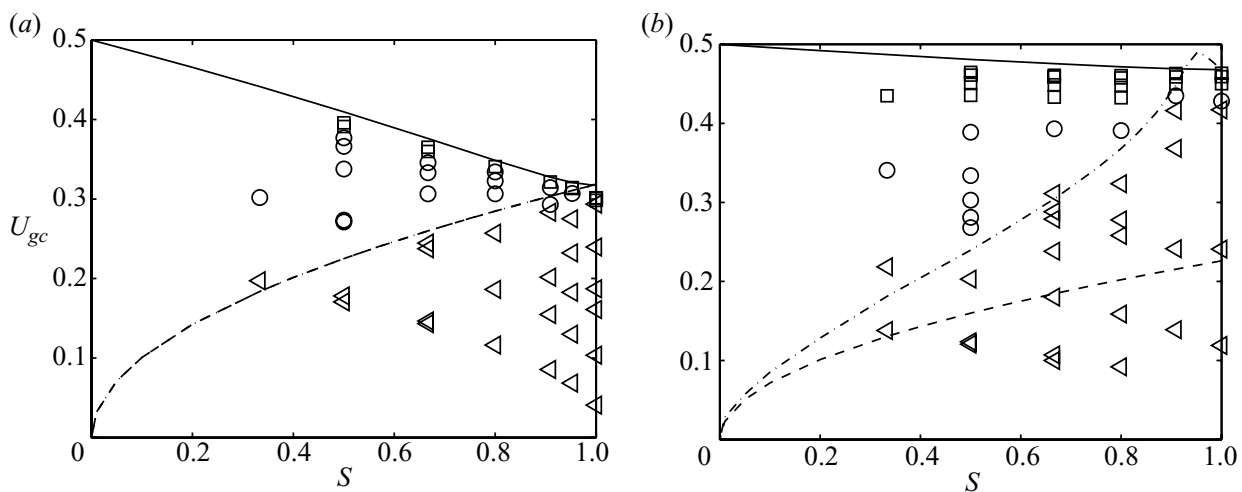

FIGURE 13. Regime diagrams in $U_{g c}$ vs. $S$ for $(a) \lambda=0$ and $(b) \lambda=8$. Bounds from steady theory are $U / c_{o}=1$ (dashed) and the upstream generation limit $\left(U / c_{l}=1\right)$ (dash-dotted), and the conjugate-state solution, $U_{g c}=U_{C S} \sqrt{S}$ (solid). Dashed and dash-dotted lines overlap for $\lambda=0$. Symbols represent the three regimes observed in the numerical results: upstream propagating waves (triangle), trapped solitary wave (circle), and steady gravity current (square).

line) approximately defines the transition between unsteady generated waves and steady flow with a single trapped wave. Note that for $\lambda=0$ (and only for this case) $c_{o}=c_{l}$, and the two curves $F r=1$ and the $U=c_{l}$ are identical. In the supercritical domain, the numerical solutions approach the conjugate state $U=U_{C S}$, but do not exceed it.

For $\lambda=8$ (figure $13 b$ ), wave-generating solutions lie within a band around $F r=1$. However, the curve $U / c_{l}=1$ appears to define the transition between unsteady wavegenerating and steadily propagating gravity currents. The numerical results give waves of very large amplitude between the $F r=1$ and $U / c_{l}=1$ curves. This is consistent with the idea of transcritical resonant forcing. In the region between these two lines, the steady theory predicts that long waves propagating above the gravity current are faster than the current, $c_{l}>U$. However, long waves in the ambient fluid ahead of the gravity current are slower, $U>c_{o}$. Thus, it is hypothesized that energy can accumulate near the gravity current front in the form of large internal waves. The speed of these highly nonlinear waves exceeds both $c_{o}$ and $U$, and they are able to outrun the gravity current. As noted for $\lambda=0, c_{o}=c_{l}$, and upstream waves occur only for $F r \leqslant 1$. Again, gravity currents for $\lambda=8$ (figure $13 b$ ) approach the conjugate-state limit for large $U$ (corresponding to large $h_{o}$ ).

Figure 14 shows a comparison between the steady gravity current theory and the numerical calculations plotted in $\mathrm{Fr}-h$ space. $\mathrm{Fr}-h$ plots organize regime transitions in Long's model and topographic resonance problems, and are relevant since we treat the current as topography of height $h$ (though, as noted, $U$ and $h$ are not decoupled). For $\lambda=0$ (figure 14a), all solutions above $F r=1$ are steady, consistent with supercritical Long's model solutions. Long's model also predicts subcritical solutions with higher vertical mode numbers within the three-humped curve shown (first three modes), but the dam-break calculations produce only wave-generating solutions within this region. These violate the steady assumptions of Long's theory and suggest Long's subcritical solutions cannot be realized from the gravity-current initial-value problem. Note that these subcritical solutions correspond to the higher mode solutions of Ungarish (2006). For $\lambda=8$ (figure 14b), no subcritical Long's solutions exist, so only the critical lines $F r=1$ (solid) and $U / c_{l}=1$ (dashed) are shown in figure $15(b)$. Internal wave solutions are found in a region around $F r=1$, 

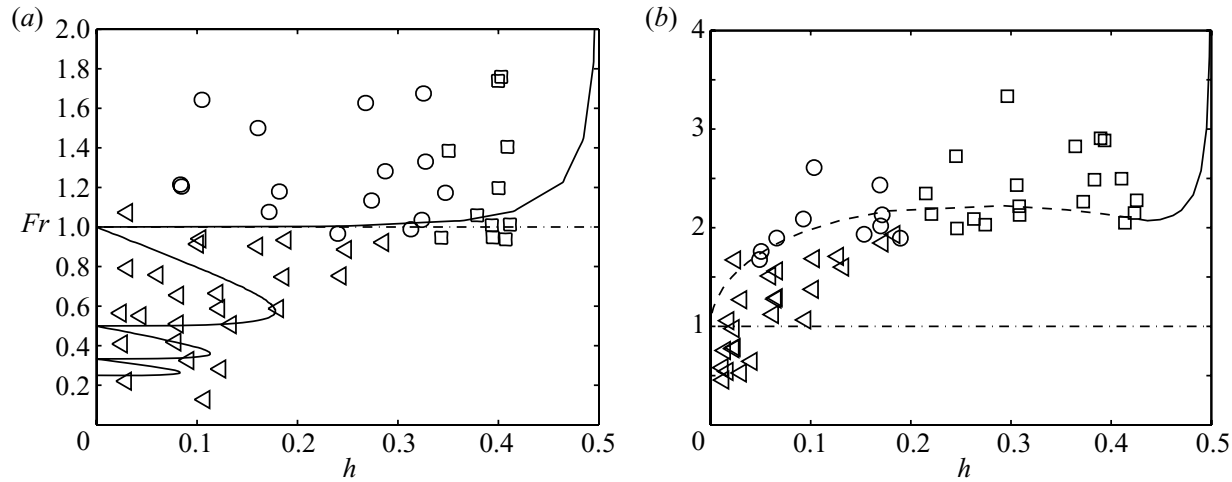

FiguRE 14. Regime diagrams in $F r$ vs. $h$. (a) $\lambda=0 . \quad F r=1$ (dash-dotted line) separates supercritical and subcritical domains. The uppermost solid line $(F r \geqslant 1)$ is the conjugate-state curve (for all $S$ between 0 and 1), the dash-dotted line marks $\mathrm{Fr}=1$. The three-humped solid line gives Long's solutions for the first three subcritical modes. $(b) \lambda=8$. Conjugate state (solid line), $F r=1$ (dash-dotted line), $U=c_{l}$ (dotted line). Symbols represent numerical solutions as in figure 13.

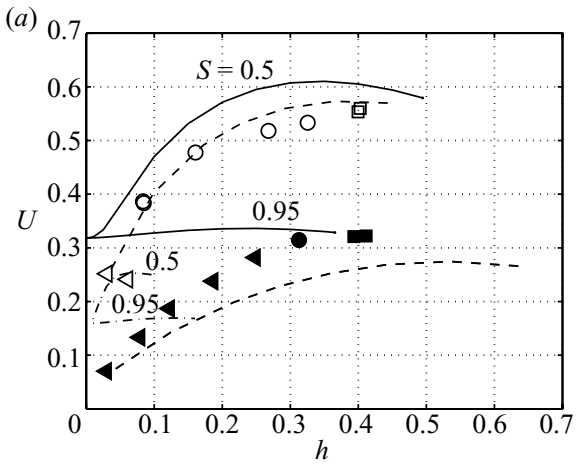

(b)
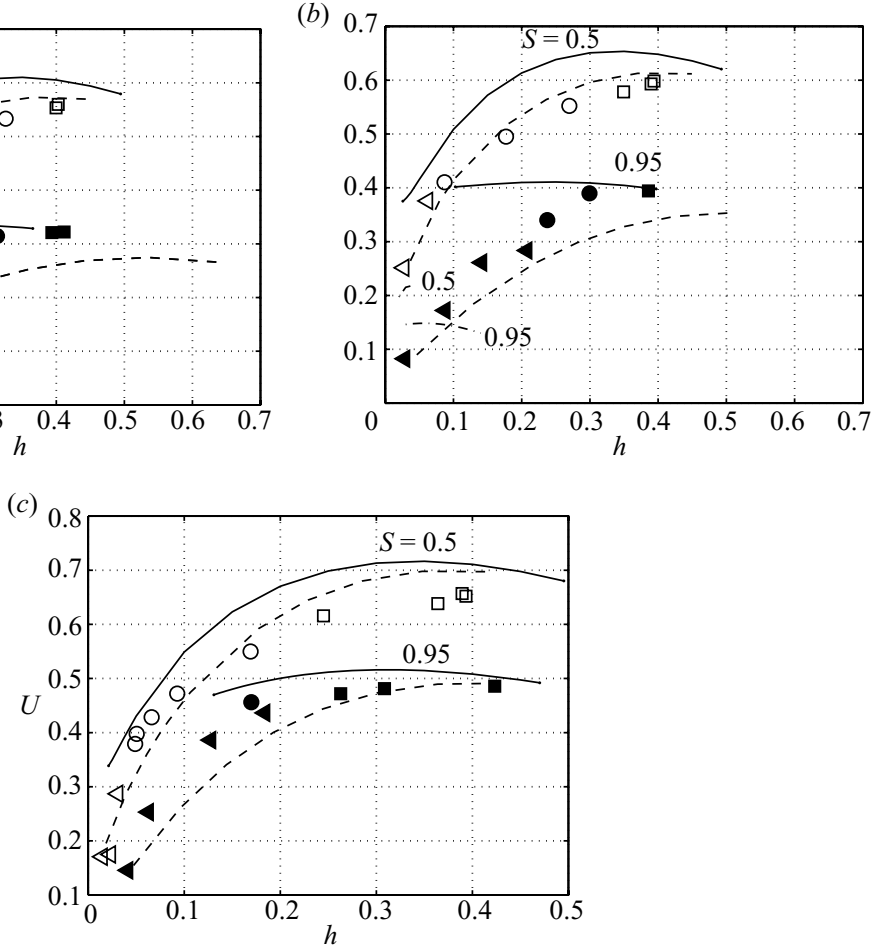

FIGURE 15. Gravity-current speed $v$ s. height for $S=0.95$ and $S=0.5$ : steady theory first root (solid lines) and second root (chain dotted lines), hydraulic theory (dashed lines) and numerical dam-break calculations (symbols, corresponding to regimes as in figures 13-14 ). (a) $\lambda=0$. (b) $\lambda=2$. (c) $\lambda=8$. Steady first-root lines bounded by the critical point at the lower terminus and the conjugate state at the upper terminus. 
consistent with topographic resonant forcing, and $U / c_{l}=1$ provides the cutoff above which only steady gravity currents are produced.

These results clarify an earlier hypothesis regarding the internal wave generation by gravity currents. Maxworthy et al. (2002) concluded that waves are associated with subcritical conditions, $F r<1$, for $\lambda=0$. The results of our theory and simulations for nonlinear stratification demonstrate that $U / c_{l} \approx 1$ divides wave-generating and steady gravity-current solutions (also the case for an intermediate stratification, $\lambda=2$ ). This is broadly consistent with 'topographic' resonance as the wave-generation mechanism. The conditions $U / c_{l}=1$ and $F r=1$ coincide only for linear stratification. Note that in nearly all of the unsteady cases, the waves generated near the front are nonlinear and travel faster than $c_{l}$. However, $c_{l}$ is the minimum speed at which waves can propagate ahead of the front, and thus $U / c_{l}$ is the appropriate 'Froude' number condition for the boundary between steady and unsteady solutions.

\subsection{Front speed: theory vs. numerical calculations}

Figure 15 depicts gravity-current speed, $U$, as a function of thickness, $h$, for $\lambda=0$, $\lambda=2$ and $\lambda=8$ for two different values of $S$. The numerical dam-break calculations are compared with the steady theory. Additionally, where they exist, the second-mode solutions described in $\S 4.3$ are shown. Also shown are dam-break solutions computed using the single-layer shallow-water hydraulic model of Ungarish \& Huppert (2002) and Ungarish (2005a) to predict both $U$ and $h$ from the dam-break initial conditions. Here, we use Benjamin's Froude-number condition $\left(U_{g c} / \sqrt{h}\right.$ from (4.9)) (see also Ungarish 2008) for the model because it gives the best agreement with our numerical dam-break calculations.

The agreement between the steady theory and the numerical dam-break results is best for smaller $S$ and for highly nonlinear stratification, $\lambda=8$. For small $S$ and large $\lambda$, the steady theory is closest to the Benjamin theory (see figures 5 and 6 ), suggesting ambient stratification plays only a small role. On the other hand, for $S \approx 1$ and $\lambda \rightarrow 0$, stratification modifies the front speed significantly. In these cases, the dam-break gravity-current speeds are well below the steady theory for subcritical conditions, $U<c_{l}$, but do approach the conjugate state (upper terminus of the curve) when $h_{o}$ is large.

Birman et al. (2007) suggest the higher-mode solutions explain slower gravitycurrent speeds in the subcritical regime and that currents released from a dam-break will lock onto the slowest stable mode. For the stratification profiles studied here, the higher-mode solutions exist for some combinations of $(h, S)$ for $\lambda=0$ and $\lambda=2$, but none could be found for $\lambda=8$. When they do exist, it is generally only for a narrow range of $h$ (figure $15 a, b$ ). It does not appear that these solutions explain the subcritical gravity current speeds sufficiently. Moreover, unsteady waves are generated in the $(h, U)$ range in which these solutions appear, which is a violation of the steady assumption of Long's theory.

The shallow-water hydraulic theory provides a much better description of current properties in the subcritical range. Both gravity-current height and speed from dambreak initial conditions are predicted surprisingly well across the full range of $(S, \lambda)$, even though the hydraulic theory neglects dynamics of isopycnal displacement in the ambient fluid. Thus, it appears that gravity-current speed and height are not substantially modified by internal-wave generation. This is consistent with results of Ungarish \& Huppert (2002), which show little energy loss from the current to internal waves. 
If current speed and height are not overly sensitive to isopycnal dynamics, as the hydraulic theory suggests, then second-mode solutions would appear to be of secondary importance. The success of the hydraulic theory suggests that the speed and height, even for subcritical currents, are set to first order by the local pressure gradient near the front, modified slightly by the ambient stratification. Once the speed is set, the details of the isopycnal displacement would be determined by Long's model dynamics (but with unsteadiness). For slower subcritical currents, the result would probably be a mode- 2 isopycnal structure, like that observed in figure 11(a).

For supercritical currents, the flow is steady, and thus within the range of validity for Long's theory. As a result, the agreement between the steady theory and the dam-break numerical results is excellent, and for some cases $(S=0.95, \lambda=0,2)$ slightly better than the hydraulic model. The best method for predicting gravity-current behaviour following dam break would appear to be some combination of the shallow-water hydraulic model and the steady Long's theory. If gravity current behaviour alone is sought, the hydraulic model gives good results. If internal-wave generation is of interest, the hydraulic model can indicate whether a current released from a given dam-break configuration will be sub- or supercritical, and Long's theory and the regime plots (figures 13-14) indicate the wave characteristics and the approach to the conjugate state.

\subsection{Available potential energy}

The approach to the conjugate-state solution as $h_{o}$ is increased, suggests that the initial available potential energy (APE) of the dammed fluid is important. The initial APE per unit length of the fluid in the lock is

$$
A P E=\int_{0}^{h_{o}}\left(\frac{1}{S}-\widetilde{\rho}(z)\right) z \mathrm{~d} z .
$$

The non-dimensionalization is $A P E=A P E^{*} /\left(\rho_{t} g^{\prime} H\right)$. The $A P E$, which is proportional to $h_{o}$ and increases with $S^{-1}$, is not a conserved quantity in this case, but is a heuristic that captures the influence of both dam height and density on the energy of the current.

The energy per unit length contained in the conjugate state is

$$
\begin{aligned}
E_{C S}=\int_{0}^{h_{C S}}\left(\frac{1}{S}-\widetilde{\rho}(z)\right) z \mathrm{~d} z & +\int_{h_{C S}}^{1}(\widetilde{\rho}(z-\eta)-\widetilde{\rho}(z)) z \mathrm{~d} z \\
& +\frac{1}{2} U_{C S}^{2} \int_{h_{C S}}^{1}\left(\left(1-\eta^{\prime}(z)\right)^{2}-1\right) \mathrm{d} z .
\end{aligned}
$$

This is the sum of potential energy in the current relative to the ambient (first two integrals) and the kinetic energy (third integral). Figure 16 shows $U / U_{C S}$ as a function of $A P E / E_{C S}$. As $A P E \rightarrow E_{C S}, U \rightarrow U_{C S}$. For $\lambda=0$ (figure 16a), there is some spread for $A P E / E_{C S}<1$, but the curves collapse for $S<0.9$. For $\lambda=8$ (figure $16 b$ ), there is good collapse for all $S$. All wave-generating solutions are confined to $A P E / E_{C S}<1$. Results for an intermediate stratification $(\lambda=2)$ are similar to $\lambda=8$ results.

\subsection{Velocity and density structure}

The density and velocity structure of the steady numerical gravity currents are compared with the corresponding conjugate-state predictions in figure 17 for $\lambda=0$ and $\lambda=8$. A vertical transect is taken through the numerical solution, including the current and the fluid above it, at the maximum of the $\rho=\left(\rho_{c}+\rho_{b}\right) / 2$ contour (where $h$ 

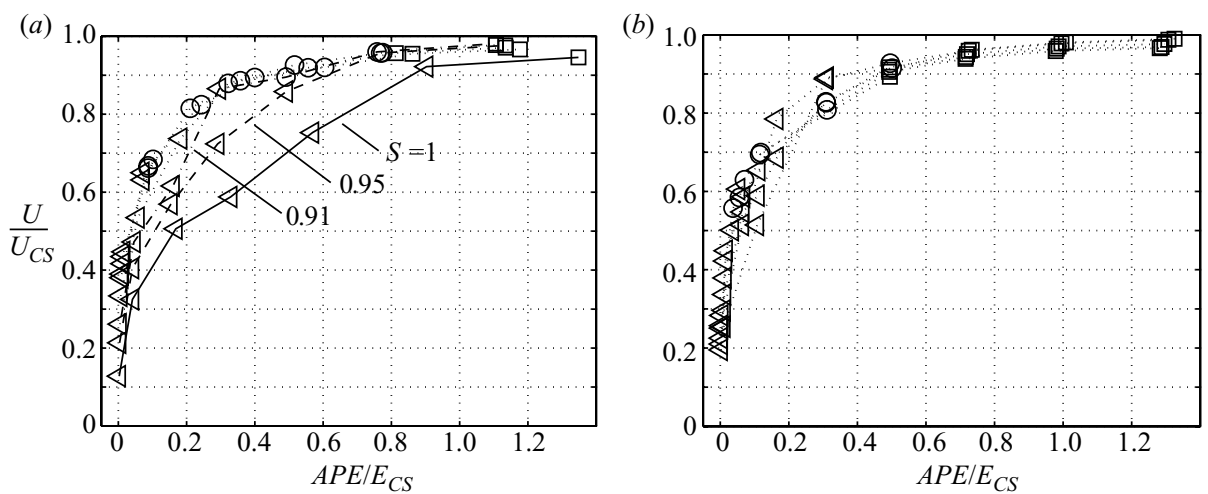

FIGURE 16. Gravity-current speed with increasing lock available potential energy (APE). (a) $\lambda=0$ (all runs across the $S$ and $h_{o}$ parameter range). (b) $\lambda=8$. Symbols represent wave-gravity-current regimes as in figures 13-14.
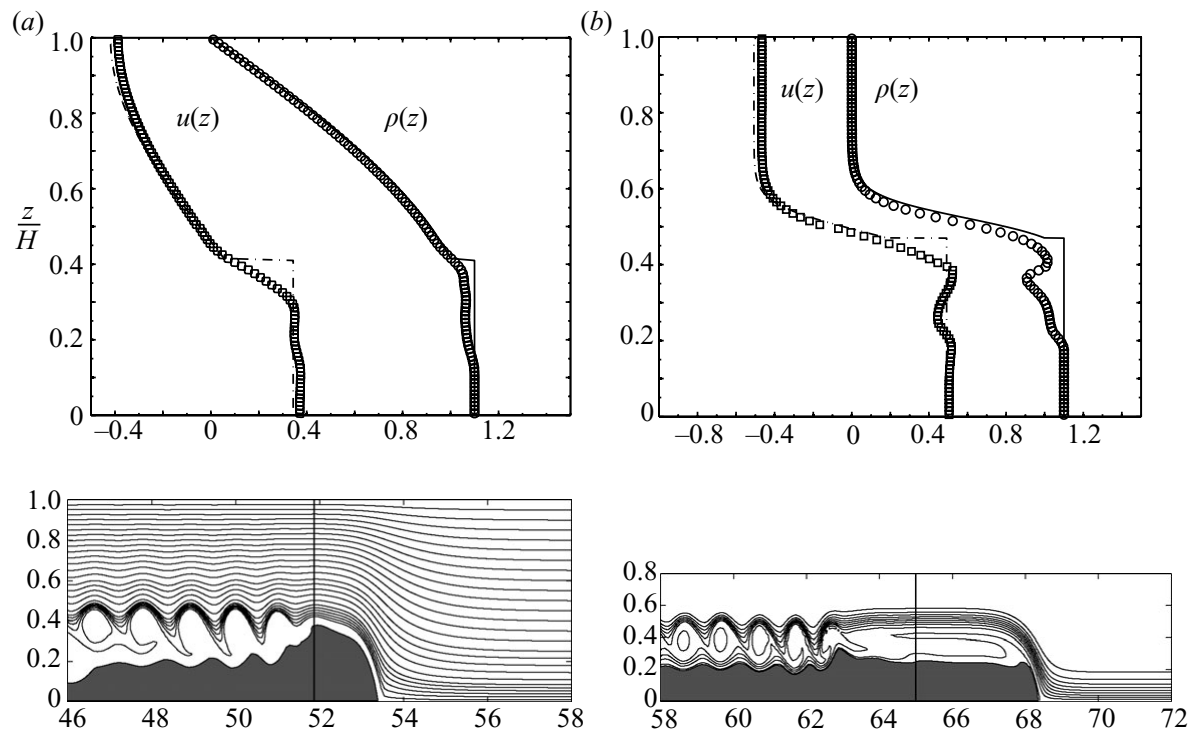

FIGURE 17. Comparison between conjugate-state solutions and numerical simulations for density and velocity profiles through the gravity current. $(a) \lambda=0, S=0.91$ : conjugate-state theory for $\rho(z)$ (solid line) and $u(z)$ (dash-dotted line) compared with vertical transects (open circles and squares, respectively) from the numerical simulations taken near the gravity current head (vertical line in inset below). (b) $\lambda=8, S=0.91$.

is defined). The agreement between the theory and the simulation results is excellent. The theory predicts a discrete density and velocity jump between the current and the fluid above, whereas in the numerical calculation, this transition is smoother owing to mixing. Because of the strong shear at the top of the current, the flow is often unstable to Kelvin-Helmholtz instabilities, which are apparent in the numerical simulations (including those shown in figure 17), and are responsible for the mixing between the current and the ambient fluid. Because of the mixing, $h$, determined as above from the numerical calculation, can be less than the conjugate-state thickness, $h_{C S}$. However, the conjugate-state theory accurately predicts the gravity-current speed as well the density and velocity structure through most of the depth. 


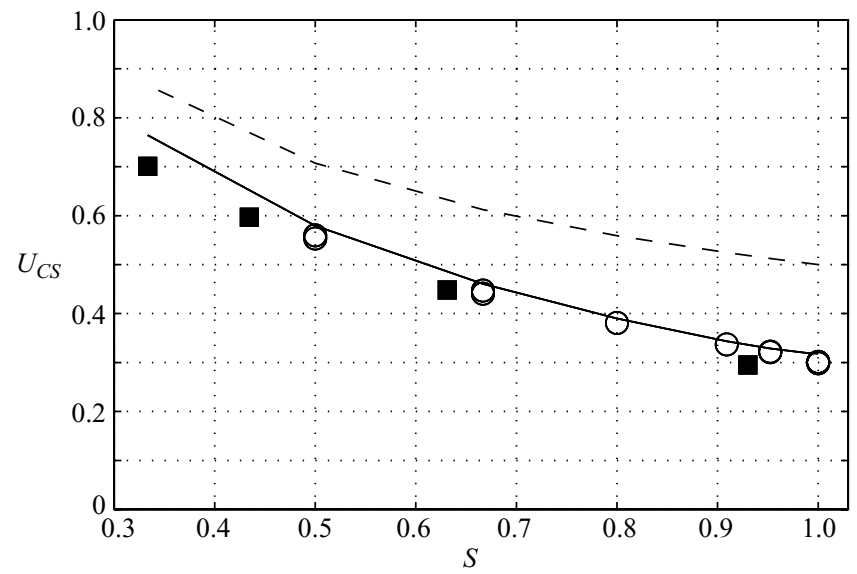

Figure 18. Comparison between the full-depth lock releases of Maxworthy et al. (2002) in a linearly stratified ambient (squares), the conjugate state wave speed predicted by the steady gravity-current theory for $\lambda=0$ (line), and the numerical results for $\lambda=0$ (open circles). Also shown for reference is the dissipationless Benjamin solution, $U_{g c}=U \sqrt{S}=0.5$ (dashed line).

\section{Comparison with existing experiments: gravity currents and intrusions}

In this section, the conjugate-state theory is compared with experiments from the literature on gravity currents and intrusions in stratified fluids. Experimental results for gravity currents generated by full-depth lock releases that do not exhibit finite domain effects are chosen for comparison. Few experiments in stratified fluids meet these requirements, but data sets are available, one from experiments by Maxworthy et al. (2002) on full-depth lock releases into a linearly stratified fluid, and experiments on neutral density $(S=1)$, symmetric intrusions by Faust \& Plate (1984) and Britter \& Simpson (1981).

\subsection{Full-depth lock releases in a linearly stratified ambient by Maxworthy et al.}

The data of Maxworthy et al. (2002) have been discussed in the context of internalwave generation, and our numerical calculations produced upstream waves under the same conditions as their experiments. In figure 18, we compare their measurements of gravity-current front speed, $U$, for full-depth lock releases, over a range of core densities, $S$, with the $\lambda=0$ conjugate-state gravity-current speed, $U_{C S}$. Also shown for comparison are our numerical results in this range, as well as the Benjamin energyconserving half-depth solution. The experimental data and the numerical results agree very well with the theoretical conjugate-state curve. The experimental front speeds consistently lie just below $U_{C S}$, probably becouse of a combination of losses due to mixing above the gravity current and friction at the no-slip bottom. The numerical simulations agree slightly better, presumably because the bottom is free-slip. The Benjamin solution significantly overpredicts the current speed. The continuously stratified result approaches the Benjamin solution in the limit $S \rightarrow 0$.

\subsection{Intrusions}

Several data sets are available for symmetric intrusions, in which a region of neutral density fluid propagates into a stratified fluid with a density profile that is symmetric about the intrusion centreline (see figure 19). If the density of the intrusion is equal to $\rho_{b}$, the mean ambient density, and the layer depths, $H$, above and below the intrusion are equal, then the intrusion is equivalent to the $S=1$ gravity-currents considered 


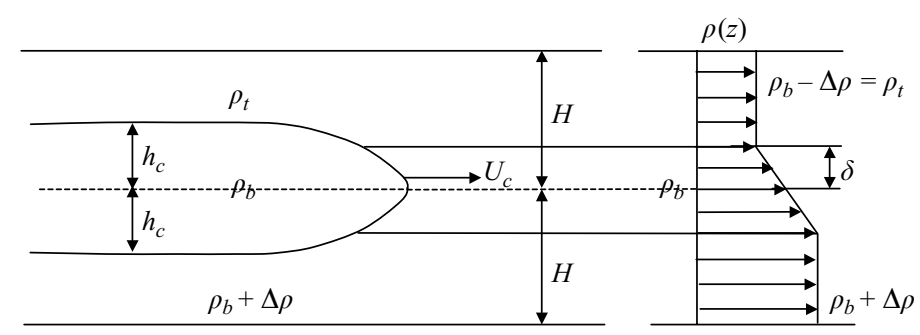

FIGURE 19. Intrusive gravity-current in a stratified ambient. The ambient is stably stratified with an overall density difference of $2 \Delta \rho$ between the uniform top and bottom layers, with an interface of thickness $\delta$ between them. If the density of the intrusion is equal to $\rho_{b}$, the mean ambient density, and the layer depths, $H$, above and below the intrusion are equal, then the intrusion may be considered equivalent to the gravity currents considered here with $S=1$.

here. The piecewise linear profile shown in figure 19 is an idealization of the density structure that results when the interface between a two-layer fluid has finite thickness $2 \delta$. These profiles, studied in the experiments of Faust \& Plate (1984) and Britter \& Simpson (1981), are best fit by an antisymmetric hyperbolic tangent profile, which is equivalent to (5.2) with reflection about $z=0$ and $\delta / H=\tanh \lambda / \lambda$. The limit $\delta / H \rightarrow 0$ represents an intrusion propagating into a two-layer fluid $(\lambda \rightarrow \infty)$, whereas the limit $\delta / H \rightarrow 1$ is an intrusion into a linearly stratified $(\lambda=0)$ fluid.

Experimental data for intrusion propagation speed and thickness from the experiments of Faust \& Plate (1984) and Britter \& Simpson (1981) are compared with the conjugate-state theory with $S=1$ in figure 20 . For both experiments, intrusions were generated by the release of a lock with height comparable to $H$, and long enough that the intrusion was not influenced by the length of the lock or domain. The agreement between the theoretical prediction and the experiments is excellent (figure 20a). In the two-layer limit $\delta / H=0$, the data, in agreement with the theory, approach the energy-conserving Benjamin solution, $F r=0.5, h=0.5$, (4.9). In the linearly stratified limit, $\delta / H=1$, the speed approaches the linear long-wave speed, $\mathrm{Fr}=1 / \pi$.

The intrusion thickness (figure 20b) is also predicted well by the steady theory. However, the linearly stratified limit is a degenerate case, as the conjugate-state theory predicts a vanishing intrusion thickness $h \rightarrow 0$ as $\delta / H \rightarrow 1$. Faust \& Plate (1984) observed a slowly decreasing intrusion thickness for $\delta / H \geqslant 0.64$, but the experimental apparatus was not long enough to allow full adjustment of the intrusion. Hence, the interface thickness for experiments with $\delta / H>0.6$ are not shown in figure $20(b)$.

\section{Conclusions}

Theory has been presented for steady gravity currents propagating in a stratified ambient with a general density profile. The theory predicts steady solutions only for a range of gravity-current thicknesses and front propagation speeds. When the front speed is less than the lower limit for steady solutions, we have shown that large-amplitude internal waves are generated at the gravity-current front and propagate ahead of the gravity current. Comparison between the theory and numerical calculations of the dam-break initial-value problem shows that the theory does an excellent job delineating the boundary between the wave-generating and the steadygravity-current solutions. Previous observations of waves excited in an ambient waveguide by a propagating density current (Maxworthy et al. 2002; Nash \& Moum 

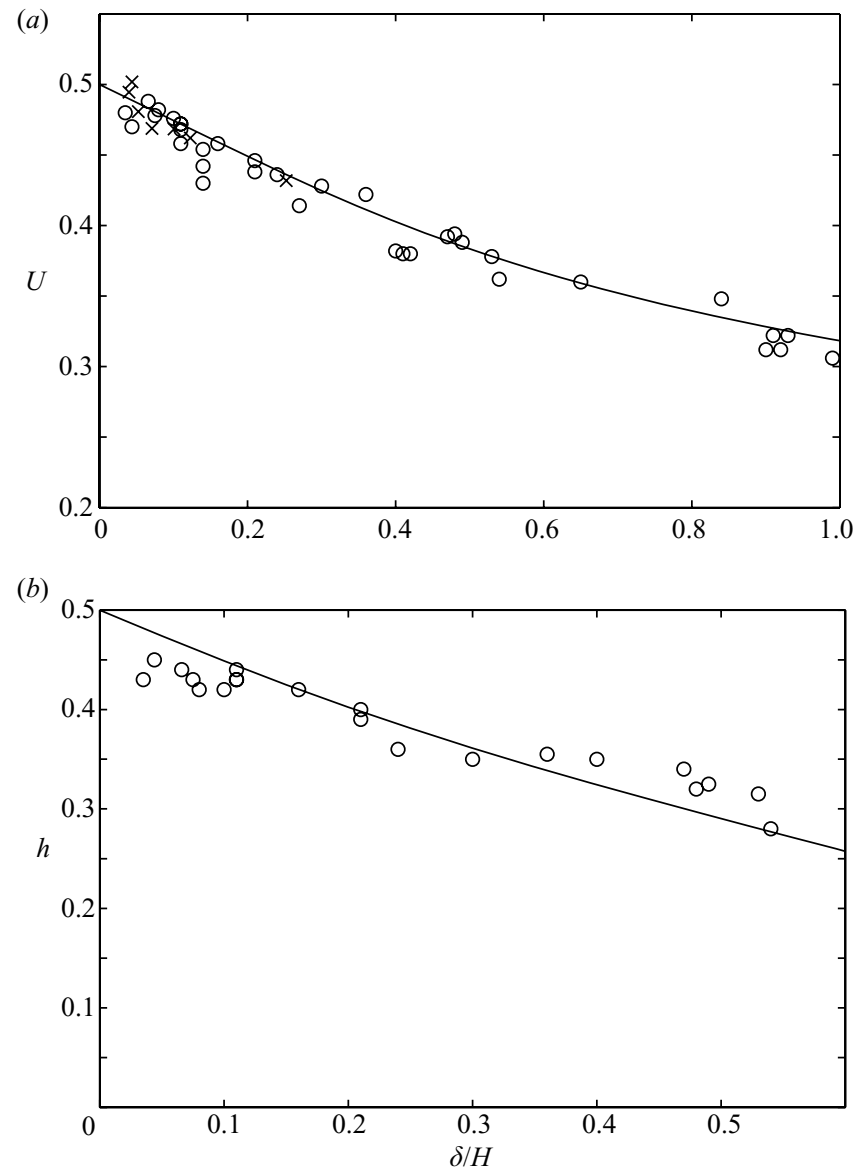

FiguRE 20. Comparison between experimental results for intrusive gravity currents and the conjugate-state theory for $S=1$ as a function of interface thickness. (a) Propagation speed of the intrusion front, O, Faust \& Plate (1984); $\times$, Britter \& Simpson (1981); - , conjugate-state speed, $S=1, \delta / H=\tanh \lambda / \lambda$. (b) Thickness of the intrusion. $\bigcirc$, experiments of Faust \& Plate (1984);--, conjugate state height.

2005) led to the hypothesis that waves exist when the gravity-current speed is less than the linear long-wave speed, $F r=U / c_{o}<1$. Rather, the theory coupled with the numerical results demonstrates that for general stratification, steady gravity currents exist only when $U>c_{l}$, the long-wave speed for waves above the gravity current. For a range of speeds, $c_{o}<U<c_{l}$, large-amplitude waves are produced, broadly consistent with a mechanism of resonant forcing over topography. Since $c_{o}=c_{l}$ for $\lambda=0$, generation of waves for $F r>1$ occurs only in nonlinear stratification $(\lambda \neq 0)$.

For sufficiently large dam height, or potential energy, gravity currents attain a limiting, supercritical form that is described well by the steady energy-conserving conjugate-state solution for the corresponding $(\lambda, S)$. The conjugate state is an extension of Benjamin's energy-conserving half-depth solution to arbitrary stratification, but is also equivalent to the limiting large-amplitude internal solitary wave with a trapped core for the given stratification. The conjugate state provides a good description of previous numerical and experimental results for gravity currents and intrusions generated by full-depth lock release. 
The steady theory is an extension of the constant $N$ results of Ungarish (2006) to arbitrary nonlinear stratification (although numerical solution is required, whereas Ungarish provided an analytical approximation for constant $N$, small $S$ ). Despite the success of the steady approaches in delineating the boundary between wavegenerating and steady solutions, and predicting the limiting energy-conserving gravitycurrent form, they describe poorly the behaviour of subcritical gravity currents, whose speeds are well below Long's theory for a given current height. The poor agreement is probably because the subcritical currents do not conform to the steady requirements of Long's theory. We explored the possibility that higher mode (slower) solutions suggested by Ungarish (2006) and Birman et al. (2007) may explain the subcritical speeds. However, higher modes are found only in a limited range of $(h, S, \lambda)$, and therefore, cannot explain all subcritical currents. Moreover, the shallowwater hydraulic theory of Ungarish \& Huppert (2002), which does not consider isopycnal displacement, does an excellent job of predicting subcritical current speeds. Thus, although the higher-mode solutions are an interesting feature of the steady solutions, they do not appear to be a useful predictive tool. A good approach for predicting the behaviour of dam-break gravity currents is to use the shallowwater model to determine the approximate current speed, after which the steady theory presented here can be used to delineate internal-wave-generating from steady solutions, and to predict the limiting energy-conserving gravity-current form for large dam height.

The class of stratifications considered have finite-density gradients at $z=0$, which is a requirement for solitary waves with trapped cores. When there is a region of constant density near the bottom, conjugate states are possible that do not reach the overturning limit and do not form trapped cores (Lamb 2002). Without the trapped core, these conjugate states are not equivalent to a gravity current, and gravity-current solutions to the dam-break problem can be expected to be fundamentally different. For these stratifications, we might expect that a full-depth lock-release may excite a bore-like conjugate state that moves faster than the gravity current. These conditions will be explored in a future paper.

This work was done while B. L. W was a Postdoctoral Scholar at the Woods Hole Oceanographic Institution. K. R. H was supported by ONR grant N000140610798.

\section{Appendix}

The flow-force balance (4.7),

$$
\int_{h}^{1}\left[p(z)-\bar{p}(z-\eta)\left(1-\eta^{\prime}\right)-U^{2} \eta^{\prime}\left(1-\eta^{\prime}\right)\right] \mathrm{d} z+\int_{0}^{h}\left[\bar{p}(0)+\frac{1}{2} \rho_{b} U^{2}-\frac{\rho_{c}}{\rho_{b}-\rho_{t}} z\right] \mathrm{d} z=0,
$$

can be simplified as follows. Consider the first two terms, which can be simplified using (4.1) and (4.5) to

$$
p(z)-\bar{p}(z-\eta)=\frac{1}{2} \rho_{b} U^{2}-\frac{\rho_{c}}{\rho_{b}-\rho_{t}} h-\int_{h}^{z} \frac{\bar{\rho}(z-\eta)}{\rho_{b}-\rho_{t}} \eta^{\prime} \mathrm{d} z .
$$

Integrating by parts, and using $N^{2}=-\mathrm{d} \bar{\rho}(z-\eta) / \mathrm{d}(z-\eta)$ and $\bar{\rho}(0)=\rho_{b}$, the integral in (A 2) can be written

$$
\int_{h}^{z} \frac{\bar{\rho}\left(z^{\prime}-\eta\right)}{\rho_{b}-\rho_{t}} \eta^{\prime} \mathrm{d} z^{\prime}=\frac{\bar{\rho}\left(z^{\prime}-\eta\right)}{\rho_{b}-\rho_{t}} \eta-\frac{\rho_{b}}{\rho_{b}-\rho_{t}} h+\int_{h}^{z} \frac{\rho_{t}}{\rho_{b}-\rho_{t}} N^{2} \eta\left(1-\eta^{\prime}\right) \mathrm{d} z^{\prime} .
$$


Then, applying the DJL equation over the region of integration, $[h, 1]$, gives $N^{2} \eta=-$ $U^{2} \eta^{\prime \prime}$, and integrating again by parts, the last integral in (A 3) can be simplified as

$$
\int_{h}^{z} \frac{\rho_{t}}{\rho_{b}-\rho_{t}} N^{2} \eta\left(1-\eta^{\prime}\right) \mathrm{d} z^{\prime}=-\frac{\rho_{t}}{\rho_{b}-\rho_{t}} U^{2}\left[\left(\eta^{\prime}\left(z^{\prime}\right)-\eta^{\prime}(h)-\frac{1}{2}\left(\eta^{\prime}\left(z^{\prime}\right)^{2}-\eta^{\prime}(h)^{2}\right)\right)\right] \text {. }
$$

Now consider the third term in the first integral of (A 1), which can be simplified by integration by parts,

$$
\int_{h}^{1} \bar{p}(z-\eta) \eta^{\prime} \mathrm{d} z=-\bar{p}(0) h+\int_{h}^{1} \frac{\bar{\rho}(z-\eta)}{\rho_{b}-\rho_{t}} \eta\left(1-\eta^{\prime}\right) \mathrm{d} z
$$

where we have taken $\bar{p}(1)=0$. Expansion and further integration by parts of the integral on the right-hand side gives

$$
\begin{aligned}
\int_{h}^{1} \bar{p}(z-\eta) \eta^{\prime} \mathrm{d} z=-\bar{p}(0) h & +\int_{h}^{1} \frac{\bar{\rho}(z-\eta)}{\rho_{b}-\rho_{t}} \eta \mathrm{d} z \\
& +\frac{\rho_{b}}{2\left(\rho_{b}-\rho_{t}\right)} h^{2}-\int_{h}^{1} \frac{1}{2\left(\rho_{b}-\rho_{t}\right)} N^{2} \eta^{2}\left(1-\eta^{\prime}\right) \mathrm{d} z
\end{aligned}
$$

Use of the DJL equation and further integration by parts simplifies the last integral in (A 6) to

$$
\begin{aligned}
\int_{h}^{1} \frac{1}{2\left(\rho_{b}-\rho_{t}\right)} N^{2} \eta^{2}\left(1-\eta^{\prime}\right) \mathrm{d} z=\frac{1}{2\left(\rho_{b}-\rho_{t}\right)}\left[U^{2} h\left(\eta^{\prime}(h)-\frac{1}{2} \eta^{\prime}(h)^{2}\right)\right. \\
\left.\quad+\int_{h}^{1} U^{2} \eta^{\prime 2} \mathrm{~d} z-\int_{h}^{1} \frac{1}{2} U^{2} \eta^{\prime 3} \mathrm{~d} z\right] .
\end{aligned}
$$

In addition, the second integral of (A 1) can be evaluated as

$$
\int_{0}^{h}\left[\bar{p}(0)+\frac{1}{2} \rho_{b} U^{2}-\frac{\rho_{c}}{\rho_{b}-\rho_{t}} z\right] \mathrm{d} z=\bar{p}(0) h+\frac{1}{2} \rho_{b} U^{2} h-\frac{1}{2} \frac{\rho_{c}}{\rho_{b}-\rho_{t}} h^{2} .
$$

Finally, substituting (A 2)-(A 8) into (A 1) and carrying out the remaining simple integration yields the result in (4.8)

$$
\int_{h}^{1} \eta^{\prime}(z)^{3} \mathrm{~d} z-\frac{1-S}{S} \frac{h(2-h)}{\frac{1}{2} U^{2}}+\eta^{\prime}(h)^{2}(2-h)-2 \eta^{\prime}(h)(2-h)+2=0 .
$$

\section{REFERENCES}

Afanasyev, Y. D. \& Peltier, W. R. 1998 The three-dimensionalization of stratified flow over a two-dimensional ridge. J. Atmos. Sci. 55, 19-39.

Baines, P. G. 1997 Topographic Effects in Stratified Flows. Cambridge University Press.

Bell, J. B. \& Marcus, D. L. 1992 A second-order projection method for variable-density flows. J. Comput. Phys. 101, 334-348.

Benjamin, T. B. 1966 Internal waves of finite amplitude and permanent form. J. Fluid Mech 25, 241-270.

Benjamin, T. B. 1968 Gravity currents and related phenomena. J. Fluid Mech. 31, 209-248.

Birman, V. K., Meiburg, E. \& Ungarish, M. 2007 On gravity currents in stratified ambients. Phys. Fluids 19, 086602-10.

Britter, R. E. \& Simpson, J. E. 1981 A note on the structure of the head of an intrusive gravity current. J. Fluid Mech. 112, 459-466. 
Brown, D. J. \& Christie, D. R. 1998 Fully nonlinear solitary waves in continuously stratified incompressible Boussinesq fluids. Phys. Fluids 10, 2569-2586.

Derzho, O. G. \& Grimshaw, R. 1997 Solitary waves with a vortex core in a shallow layer of stratified fluid. Phys. Fluids 9, 3378-3385.

Faust, K. M. \& Plate, E. J. 1984 Experimental investigation of intrusive gravity currents entering stably stratified fluids. J. Hydraul. Res. 22, 315-325.

FlynN, M. R \& Linden, P. F. 2006 Intrusive gravity currents. J. Fluid Mech. 568, 193-202.

Grimshaw, R. H. J. \& SMYth, N. 1986 Resonant flow of a stratified fluid over topography. J. Fluid Mech. 169, 429-464.

Grimshaw, R. \& YI, Z. 1991 Resonant generation of finite amplitude waves by the flow of a uniformly stratified fluid over topography. J. Fluid Mech. 229, 603-628.

Helfrich, K. R. \& Melville, W. K. 2006 Long nonlinear internal waves. Annu. Rev. Fluid Mech. 38, 395-425.

Helfrich, K. R. \& Pineda, J. 2003 Accumulation of particles in propagating fronts. Limnol. Oceanogr. 48, 1509-1520.

LAMB, K. G. 2002 A numerical investigation of solitary internal waves with trapped cores formed via shoaling. J. Fluid Mech. 451, 109-144.

LAmb, K. G. \& WAN, B. G. 1998 Conjugate flows and flat solitary waves for a continuously stratified fluid. Phys. Fluids 10, 2061-2079.

Lamb, K. G. \& Wilkie, K. P. 2004 Conjugate flows for waves with trapped cores. Phys. Fluids 16, 4685-4695.

Lowe, R. J., RotTman, J. W. \& Linden, P. F. 2005 The non-Boussinesq lock-exchange problem. Part 1. Theory and experiments. J. Fluid Mech. 537, 101-124.

Margolin, L. G., Rider, W. J. \& Grinstein, F. F. 2006 Modeling turbulent flow with implicit LES. J. Turbulence 15, DOI:10.1080/14685240500331595.

Maxworthy, T., Leilich, J., Simpson, J. E. \& Meiburg, E. H. 2002 The propagation of a gravity current into a linearly stratified fluid. J. Fluid Mech. 453, 371-394.

Melville, W. K. \& Helfrich, K. R. 1987 Transcritical two-layer flow over topography. J Fluid Mech. 178, 31-52.

Miles, J. W. 1980 Solitary waves. Annu. Rev. Fluid Mech. 12, 11-43.

NASh, J. D. \& Moum, J. N. 2005 River plumes as a source of large-amplitude internal waves in the coastal ocean. Nature 437, 400-403.

PInEDA, J. 1991 Predictable upwelling and the shoreward transport of planktonic larvae by internal tidal bores. Science 253, 548-551.

Rottman, J. W. \& Simpson, J. E. 1989 The formation of internal bores in the atmosphere-a laboratory model. Q. J. R. Met. Soc. 115, 941-963.

Shin, J. O., Dalziel, S. B. \& Linden, P. F. 2004 Gravity currents produced by lock exchange. J. Fluid Mech. 521, 1-34.

Simpson, J. E. 1999 Gravity Currents: In the Environment and the Laboratory, 2nd edn. Cambridge University Press.

Ungarish, M. 2005a Dam-break release of a gravity current in a stratified ambient. Eur. J. Mech. B Fluids 24, 642-658.

Ungarish, M. $2005 b$ Intrusive gravity currents in a stratified ambient: shallow-water theory and numerical results. J. Fluid Mech. 535, 287-323.

UNGARISH, M. 2006 On gravity currents in a linearly stratified ambient: a generalization of Benjamin's steady-state propagation results. J. Fluid Mech. 548, 49-68.

UNGARISH, M. 2008 On energy balances and front speed conditions for two-layer models for gravity currents produced by lock release. Acta Mech, doi:10.1007/S00707-008-0073-z.

Ungarish, M. \& HuPPERT, H. E. 2002 On gravity currents propagating at the base of a stratified ambient. J. Fluid Mech. 458, 283-301.

UNGARISH, M. \& HuPPERT, H. E. 2006 Energy balances for propagating gravity currents: homogeneous and stratified ambients. J. Fluid Mech. 565, 363-380. 